\title{
FATIGUE AND PHASE TRANSITION IN AN OSCILLATING ELASTOPLASTIC BEAM*
}

\author{
Michela Eleuteri ${ }^{1, * *}$, Chiara Gavioli ${ }^{1}$ and Jana Kopfová ${ }^{2}$
}

\begin{abstract}
We study a model of fatigue accumulation in an oscillating elastoplastic beam under the hypothesis that the material can partially recover by the effect of melting. The model is based on the idea that the fatigue accumulation is proportional to the dissipated energy. We prove that the system consisting of the momentum and energy balance equations, an evolution equation for the fatigue rate, and a differential inclusion for the phase dynamics admits a unique strong solution.
\end{abstract}

Mathematics Subject Classification. 47J40, 74R20, 74K10, 74N30, 82B26.

Received December 27, 2018. Accepted November 17, 2019.

\section{INTRODUCTION}

Our present paper deals with a model for the cyclic fatigue accumulation in a transversally oscillating elastoplastic beam, which is based on the idea that the fatigue accumulation is proportional to the dissipated energy. Temperature and phase transition effects, which usually accompany the process, are taken into account. The full system of equations consists of the momentum balance equation coupled with the energy balance, fatigue accumulation equation and a differential inclusion for the phase dynamics.

For our model we first use the results from [20], where by means of the Kirchhoff-Love method it was shown that the 3D problem of transversal oscillations of a solid elastoplastic beam with the single yield von Mises plasticity law can be transformed into the beam equation with a multiyield hysteresis Prandtl-Ishlinskii constitutive operator.

The simplified model was first derived in [9] and its thermodynamical consistency was shown there.

The mathematical treatment of the model started in [11], where we considered the non-isothermal case and a simplified setting - only the elastic component of the model depends on the fatigue parameter. We showed existence and uniqueness of a strong solution in a time interval depending on the size of the data.

In [4] we presented a new phase field model under the additional hypothesis that the material can partially recover by the effect of local melting. The system of equations was completed with a differential inclusion for the phase dynamics.

\footnotetext{
* Supported by the institutional support for the development of research organizations IČ 47813059 . The work of the first two authors is supported by GNAMPA (Gruppo Nazionale per l'Analisi Matematica, la Probabilità e le loro Applicazioni) of INdAM (Istituto Nazionale di Alta Matematica), by the University of Modena and Reggio Emilia through the project FAR2017 "Equazioni differenziali: problemi evolutivi, variazionali ed applicazioni" (coord. Prof. S. Gatti) and by MIUR (Ministero dell'Istruzione, dell'Università e della Ricerca) through the project FFABR2017.

${ }^{1}$ University of Modena and Reggio Emilia, Dipartimento di Scienze Fisiche, Informatiche e Matematiche,

via Campi 213/b, 41125 Modena, Italy.

2 Mathematical Institute of the Silesian University, Na Rybníčku 1, 74601 Opava, Czech Republic.

** Corresponding author: michela.eleuteri@unimore.it
} 
The mathematical treatment for the phase model for the beam started in [13], where we proved existence and uniqueness of a strong solution under the simplified assumption that the elastic component of the model is independent of the fatigue parameter while the plastic one depends only on the fatigue. We assumed proportionality of the fatigue rate to the plastic dissipation.

The $2 \mathrm{D}$ case - the plate - was considered in [10], assuming that both the elastic and the plastic characteristics change with increasing fatigue, under the simplified situation of fixed temperature. We obtained existence and uniqueness of a solution locally in time; accordingly to the model indeed, material softening takes place under increasing fatigue and material failure is manifested in finite time.

The non-isothermal 2D model has been considered in [12], where we proved existence of solutions for the whole time interval, taking a perspective usually considered in engineering analysis, assuming that the fatigue accumulation rate is proportional only to the plastic part of the dissipation rate. From the mathematical viewpoint, the problem does not exhibit singularities anymore and the expected solutions turn to be global in time.

In [14] also the dependence of the plastic dissipation on both the temperature and the fatigue parameter was considered in the $2 \mathrm{D}$ case. It was mentioned that in principle it makes sense from the point of view of modeling and applications to allow a further dependence of the Prandlt-Ishlinskii density on the phase parameter and it was shown, assuming a new special flow rule for the phase variable, that the resulting model is still thermodynamically consistent.

Here we start the mathematical analysis of this new problem, first in the $1 \mathrm{D}$ case, i.e. we additionally allow this dependence of the Prandtl-Ishlinskii density function not only on the fatigue parameter, as was first considered in [13], but also on the phase variable.

The aim of the present paper is to show existence and uniqueness of a strong solution of the underlying system of equations, this brings non-trivial mathematical difficulties.

We remark that our viewpoint is different from the contributions already present in the literature: on one hand we focus on the dynamics (compared, for instance, with $[18,26,30]$ that go into the direction of rateindependent damage processes in nonlinear elasticity and/or thermo-elasto-plasticity, see also [8, 28, 29]) and also because the approach used in other papers, that interpret damage processes as a kind of phase transition in the material (see for instance $[1-3,27]$ ), is based on the idea that damage processes are driven by large deformations. Moreover, the multiyield character emerging from our bending problem does not seem to have been taken into consideration before, neither in the multidimensional setting: see for instance [5, 7, 21, 24]. Finally the methods of the papers $[17,22,25]$ based on $\Gamma$-convergence of energy minimizers have been only recently refined in [23] to obtain the Prandtl-Ishlinskii model in the $\Gamma$-limit as well; this approach has the drawback that it cannot be extended to the study of oscillating systems, and of nonequilibrium problems in general. On the other hand, new approaches are constantly appearing in the literature to describe in the most effective way the fatigue in materials, we quote for instance [31, 33].

The plan of the paper is the following: Section 2 deals with the Prandtl-Ishlinskii model and its properties; in Section 3 we derive the model; Section 4 contains the statement of our main result and finally Sections 5-7 contain the proof of the existence part of our theorem, while Section 8 deals with the proof of the uniqueness.

\section{Hysteresis in ELASTO-PLASTICITY: THE STOP OPERATOR}

Before presenting our model problem, we would like to recall some basic facts about an important hysteresis operator which is widely used in modelling elasto-plastic processes: the Prandtl-Ishlinskii operator. Let us first recall the definition of the stop. For more details we refer for instance to [19].

Definition 2.1. Let $u \in W^{1,1}(0, T)$ and a closed interval $Z \subset \mathbb{R}$ be given. We define the stop operator $\mathfrak{s}_{Z}$ and the play operator $\mathfrak{p}_{Z}$ by the formula

$$
z(t)=\mathfrak{s}_{Z}\left[z^{0}, u\right](t), \quad \xi(t)=\mathfrak{p}_{Z}\left[z^{0}, u\right](t),
$$


where $z(t)$ is the unique solution of the variational inequality

$$
\begin{aligned}
& u(t)=z(t)+\xi(t) \quad \forall t \in[0, T], \\
& z(t) \in Z, \\
& \dot{\xi}(t)(z(t)-y) \geq 0 \quad \text { a.e. } \quad \forall y \in Z, \\
& z(0)=z^{0} \in Z .
\end{aligned}
$$

It is well known, see e.g. [19], that for each $u \in W^{1,1}(0, T)$ and each $z^{0} \in Z$ the solution $z(t) \in W^{1,1}(0, T)$ of (2.2) exists and is unique. For a canonical choice of $Z=[-r, r]$ with some $r>0$ and for the initial condition $z(0)=Q_{r}(u(0))$, where $Q_{r}$ is the projection of $\mathbb{R}$ onto the interval $[-r, r]$, we simply write

$$
z(t)=\mathfrak{s}_{r}[u](t), \quad \xi(t)=\mathfrak{p}_{r}[u](t) .
$$

A simple proof of the following easy properties of the play and stop can be found e.g. in ([19], Prop. II.1.1).

Proposition 2.2. Let $u_{1}, u_{2} \in W^{1,1}(0, T)$, a closed interval $Z \subset \mathbb{R}$, and data $z_{1}^{0}, z_{2}^{0} \in Z$ be given, $z_{i}=\mathfrak{s}_{Z}\left[z_{i}^{0}, u_{i}\right]$, $\xi_{i}=u_{i}-z_{i}, i=1,2$. Then $z_{i}, \xi_{i} \in W^{1,1}(0, T)$, and

(i) $\left(z_{1}(t)-z_{2}(t)\right)\left(\dot{u}_{1}(t)-\dot{u}_{2}(t)\right) \geq \frac{1}{2} \frac{\mathrm{d}}{\mathrm{d} t}\left(z_{1}(t)-z_{2}(t)\right)^{2} \quad$ a.e.;

(ii) $\left|\dot{\xi}_{1}(t)-\dot{\xi}_{2}(t)\right|+\frac{\mathrm{d}}{\mathrm{d} t}\left|z_{1}(t)-z_{2}(t)\right| \leq\left|\dot{u}_{1}(t)-\dot{u}_{2}(t)\right| \quad$ a.e.;

(iii) $\left|z_{1}(t)-z_{2}(t)\right| \leq\left|z_{1}^{0}-z_{2}^{0}\right|+2 \max _{0 \leq \tau \leq t}\left|u_{1}(\tau)-u_{2}(\tau)\right| \quad \forall t \in[0, T]$;

(iv) $\dot{\xi}_{i}(t) \dot{u}_{i}(t)=\dot{\xi}_{i}(t)^{2}$ a.e.

The variational inequality (2.2) is also used to model single-yield elastoplasticity. In this case, the constraint $Z=[-r, r]$ is the admissible stress domain, the input $u=\varepsilon$ is the strain, and the output $z=\sigma_{r}:=\mathfrak{s}_{r}[\varepsilon]$ is the stress. We can rewrite (2.2) equivalently in "energetic" form

$$
\dot{\varepsilon}(t) \sigma_{r}(t)=\frac{\mathrm{d}}{\mathrm{d} t}\left(\frac{1}{2} \sigma_{r}^{2}(t)\right)+r|\dot{\xi}(t)| .
$$

Indeed, $\dot{\varepsilon}(t) \sigma_{r}(t)$ is the power supplied to the system, part of it is used for the increase of the potential $\frac{1}{2} \sigma_{r}^{2}(t)$, and the rest $r|\dot{\xi}(t)|$ is dissipated.

The Prandtl-Ishlinskii model is constructed as a combination of stops with all possible yield points $r>0$. Here, given a measurable function $\varphi:[0, \infty) \times[0,1] \times(0, \infty) \rightarrow[0, \infty)$ satisfying Hypothesis 4.1 (i) below, we define the Prandtl-Ishlinskii operator, dependent on the fatigue and phase variable, $P:\left(W^{1,1}(0, T)\right)^{3} \rightarrow W^{1,1}(0, T)$ by the integral

$$
P[m, \chi, \varepsilon](t)=\int_{0}^{\infty} \varphi(m(t), \chi(t), r) \mathfrak{s}_{r}[\varepsilon](t) \mathrm{d} r
$$

Equation (2.4) enables us to establish the energy balance for the Prandtl-Ishlinskii operator (2.5). Indeed, if we define the Prandtl-Ishlinskii potential

$$
V[m, \chi, \varepsilon](t)=\frac{1}{2} \int_{0}^{\infty} \varphi(m, \chi, r) \mathfrak{s}_{r}^{2}[\varepsilon](t) \mathrm{d} r,
$$

and the dissipation operator

$$
D[m, \chi, \varepsilon](t)=\int_{0}^{\infty} r \varphi(m, \chi, r)\left|\mathfrak{p}_{r}[\varepsilon]_{t}(t)\right| \mathrm{d} r
$$


we can write the Prandtl-Ishlinskii energy balance in the form

$$
\begin{aligned}
\dot{\varepsilon}(t) P[m, \chi, \varepsilon](t)= & \frac{\mathrm{d}}{\mathrm{d} t} V[m, \chi, \varepsilon](t)+D[m, \chi, \varepsilon](t) \\
& -\frac{1}{2} m_{t} \int_{0}^{\infty} \varphi_{m}(m, \chi, r) \mathfrak{s}_{r}^{2}[\varepsilon](t) \mathrm{d} r-\frac{1}{2} \chi_{t} \int_{0}^{\infty} \varphi_{\chi}(m, \chi, r) \mathfrak{s}_{r}^{2}[\varepsilon](t) \mathrm{d} r \quad \text { a.e. }
\end{aligned}
$$

As a consequence of Proposition 2.2 (iv), we have

$$
D[m, \chi, \varepsilon](t) \leq|\dot{\varepsilon}(t)| \int_{0}^{\infty} r \varphi(m, \chi, r) \mathrm{d} r .
$$

\section{THE MODEL}

In this paper we deal with a transversally inhomogeneous beam of length 1 ; we denote by $x \in[0,1]$ the longitudinal variable, by $t \in[0, T]$ the time variable, by $w(x, t)$ the transversal displacement of the point $x$ at time $t$, by $\varepsilon(x, t)=w_{x x}(x, t)$ the linearized curvature, and by $\sigma(x, t)$ the bending moment. The new thermovisco-elasto-plastic scalar constitutive relation can be written in the form

$$
\sigma=B \varepsilon+P[m, \chi, \varepsilon]+\nu \varepsilon_{t}-\beta\left(\theta-\theta_{\text {ref }}\right),
$$

with $B>0$ the elastic modulus, $m \geq 0$ a scalar time and space dependent parameter which accounts for the accumulation of fatigue, $\nu$ the viscosity coefficient and $\beta$ the thermal expansion coefficient related to a layered structure of the beam. Moreover $\theta>0$ is the absolute temperature, $\theta_{\text {ref }}$ is the melting temperature, which is considered as a fixed referential temperature. As we already outlined the main novelty is the dependence on $\chi$ of the Prandtl-Ishlinskii constitutive operator of elasto-plasticity $P[m, \chi, \varepsilon]$, see $(2.5)$, extended in a natural way to a space continuous dependent input.

Following [20], we can write Newton's law of motion as

$$
\rho w_{t t}-\alpha w_{x x t t}+\sigma_{x x}=F(x, t) ;
$$

here $\alpha=\rho l^{2} / 12$ with $l>0$ the thickness of the beam, $\rho$ is the mass density, which is assumed to be constant, and $F$ the external load.

The following free energy operator is associated with the new constitutive law (3.1)

$$
\begin{aligned}
\mathcal{F}(m, \theta, \chi, \varepsilon)= & c \theta\left(1-\log \left(\theta / \theta_{\text {ref }}\right)\right)+\frac{B}{2} \varepsilon^{2}+V[m, \chi, \varepsilon] \\
& -\beta\left(\theta-\theta_{\text {ref }}\right) \varepsilon-\frac{L}{\theta_{\text {ref }}}\left(\theta-\theta_{\text {ref }}\right) \chi+I_{[0,1]}(\chi),
\end{aligned}
$$

where $V[m, \chi, \varepsilon]$ is the fatigue dependent Prandtl-Ishlinskii potential introduced in (2.6) while the specific heat capacity $c$ and the latent heat $L$ are given constants; moreover $I_{[0,1]}$ denotes the indicator function of the interval $[0,1]$. The entropy operator $\mathcal{S}$ and internal energy operator $\mathcal{U}$ consequently have the form

$$
\begin{aligned}
\mathcal{S}(\theta, \chi, \varepsilon) & =-\frac{\partial \mathcal{F}}{\partial \theta}=c \log \left(\theta / \theta_{\text {ref }}\right)+\beta \varepsilon+\frac{L}{\theta_{\text {ref }}} \chi, \\
\mathcal{U}(m, \theta, \chi, \varepsilon) & =\mathcal{F}(m, \theta, \chi, \varepsilon)+\theta \mathcal{S}(\theta, \chi, \varepsilon)=c \theta+\frac{B}{2} \varepsilon^{2}+V[m, \chi, \varepsilon]+\beta \theta_{\mathrm{ref}} \varepsilon+L \chi+I_{[0,1]}(\chi) .
\end{aligned}
$$

We consider the first and the second principles of thermodynamics in the form

$$
\begin{aligned}
& \mathcal{U}(m, \theta, \chi, \varepsilon)_{t}+q_{x}=\varepsilon_{t} \sigma+g, \\
& \mathcal{S}(\theta, \chi, \varepsilon)_{t}+\left(\frac{q}{\theta}\right)_{x} \geq \frac{g}{\theta}
\end{aligned}
$$


where $q=-\kappa \theta_{x}$ is the heat flux with a constant heat conductivity $\kappa>0$, and $g$ is the heat source density. Note that (3.6) is the energy conservation law, and by (2.8) it is equivalent to

$$
\begin{aligned}
c \theta_{t}-\kappa \theta_{x x} & =D\left[m, \chi, w_{x x}\right]+\nu w_{x x t}^{2}-\beta \theta w_{x x t}-\frac{1}{2} m_{t} \int_{0}^{\infty} \varphi_{m}(m, \chi, r) \mathfrak{s}_{r}^{2}\left[w_{x x}\right] \mathrm{d} r \\
& -\frac{1}{2} \chi_{t} \int_{0}^{\infty} \varphi_{\chi}(m, \chi, r) \mathfrak{s}_{r}^{2}\left[w_{x x}\right](t) \mathrm{d} r-L \chi_{t}+g(\theta, x, t) .
\end{aligned}
$$

On the other hand, (3.7) is the Clausius-Duhem inequality, and we will check in Section 4 after stating Hypothesis 4.1 that the system is thermodynamically consistent.

The evolution of the phase variable $\chi$ is assumed to be of "phase-relaxation" type, i.e. governed by the inclusion $-\gamma \chi_{t} \in \partial_{\chi} \mathcal{F}$ (see e.g. [16, 32]), that is,

$$
-\gamma \chi_{t} \in \partial I_{[0,1]}(\chi)-\frac{L}{\theta_{\text {ref }}}\left(\theta-\theta_{\text {ref }}\right)+\frac{1}{2} \int_{0}^{\infty} \varphi_{\chi}(m, \chi, r) \mathfrak{s}_{r}^{2}[\varepsilon](t) \mathrm{d} r
$$

where $\gamma>0$ is a characteristic time of phase transition and $\partial I_{[0,1]}$ is the subdifferential of the indicator function $I_{[0,1]}$. Indeed, we necessarily have $\chi \in[0,1]$, and we interpret $\chi=0$ as the solid phase, $\chi=1$ as liquid, and the intermediate values correspond to the relative liquid content in a mixture of the two.

Let $D[m, \chi, \varepsilon]$ be the dissipation operator defined in (2.7) associated with the Prandtl-Ishlinskii operator $P[m, \chi, \varepsilon]$. The analysis of the so-called rainflow method of cyclic fatigue accumulation in elastoplastic materials carried out in [6] has shown a close relation between accumulated fatigue and dissipated energy, similarly as in [15]. Here, we assume in addition that partial recovery of the damaged material is possible under strong local melting. Mathematically, this is expressed in terms of the evolution equation for the fatigue variable $m$

$$
m_{t}(x, t) \in-\partial I_{[0, \infty)}(m)-h\left(\chi_{t}(t)\right)+\int_{0}^{1} \lambda(x-y) D[m, \chi, \varepsilon](y, t) \mathrm{d} y,
$$

where $h$ is a nonnegative nondecreasing function vanishing for negative arguments, see Hypothesis 4.1 (vi), $\lambda$ is a nonnegative smooth function with (small) compact support and $D[m, \chi, \varepsilon]$ is the fatigue dependent dissipation operator, see (2.7). The subdifferential $\partial I_{[0, \infty)}$ of the indicator function $I_{[0, \infty)}$ ensures that the fatigue parameter remains nonnegative.

The meaning of (3.10) is simple. If no phase transition takes place or if the material solidifies, that is, $\chi_{t} \leq 0$, then fatigue at a point $x$ increases proportionally to the energy dissipated in a neighborhood of the point $x$. On the other hand, under strong melting if $\chi$ grows faster than the plastic dissipation rate, the fatigue may decrease until it possibly reaches the unperturbed state $m=0$.

Observing that the variational inequality $(2.2)$ can be rewritten in an equivalent way as the inclusion $\dot{z}(t)+$ $\partial I_{Z}(z(t)) \ni \dot{u}(t)$, we can interpret the differential inclusions (3.9) and (3.10) for the phase variable $\chi$ and fatigue variable $m$ with a choice $\chi^{0}(x) \in[0,1], m^{0}(x) \geq 0$ of initial conditions in an equivalent way in the form

$$
\begin{aligned}
\chi(x, t) & =\mathfrak{s}_{[0,1]}\left[\chi^{0}(x), A(x, \cdot)\right](t), \\
m(x, t) & =\mathfrak{s}_{[0, \infty)}\left[m^{0}(x), S(x, \cdot)\right](t),
\end{aligned}
$$

where

$$
\begin{aligned}
A(x, t) & :=\int_{0}^{t} \frac{1}{\gamma}\left(\frac{L}{\theta_{\text {ref }}}\left(\theta-\theta_{\text {ref }}\right)-\frac{1}{2} \int_{0}^{\infty} \varphi_{\chi}(m, \chi, r) \mathfrak{s}_{r}^{2}[\varepsilon](t) \mathrm{d} r\right)(x, \tau) \mathrm{d} \tau, \\
S(x, t) & :=\int_{0}^{t}\left(-h\left(\chi_{t}(\tau)\right)+\int_{0}^{1} \lambda(x-y) D[m, \chi, \varepsilon](y, \tau) \mathrm{d} y\right)(x, \tau) \mathrm{d} \tau .
\end{aligned}
$$

The advantage of this representation is that now, $\chi$ and $m$ are defined by equations involving, by virtue of Proposition 2.2, only operators that are Lipschitz continuous in $W^{1,1}(0, T)$. 


\section{Statement of THE PROBlem}

For any $T>0$, we set

$$
\Omega_{T}:=(0,1) \times(0, T), \quad u(x, t)=\int_{0}^{t} \sigma(x, \tau) \mathrm{d} \tau, \quad f(x, t)=\int_{0}^{t} F(x, \tau) \mathrm{d} \tau+\rho w_{t}(x, 0)-\alpha w_{x x t}(x, 0) .
$$

We rewrite the equations (3.1), (3.2), (3.8), (3.9), (3.10) as the system

$$
\begin{aligned}
u_{t}= & B w_{x x}+P\left[m, \chi, w_{x x}\right]+\nu w_{x x t}-\beta\left(\theta-\theta_{\text {ref }}\right), \\
\rho w_{t}-\alpha w_{x x t}= & -u_{x x}+f(x, t), \\
c \theta_{t}-\kappa \theta_{x x}= & D\left[m, \chi, w_{x x}\right]+\nu w_{x x t}^{2}-\beta \theta w_{x x t}-\frac{1}{2} m_{t} \int_{0}^{\infty} \varphi_{m}(m, \chi, r) \mathfrak{s}_{r}^{2}\left[w_{x x}\right] \mathrm{d} r \\
& -\frac{1}{2} \chi_{t} \int_{0}^{\infty} \varphi_{\chi}(m, \chi, r) \mathfrak{s}_{r}^{2}\left[w_{x x}\right](t) \mathrm{d} r-L \chi_{t}+g(\theta, x, t), \\
-\gamma \chi_{t} \in & \partial I_{[0,1]}(\chi)-\frac{L}{\theta_{\text {ref }}}\left(\theta-\theta_{\text {ref }}\right)+\frac{1}{2} \int_{0}^{\infty} \varphi_{\chi}(m, \chi, r) \mathfrak{s}_{r}^{2}\left[w_{x x}\right](t) \mathrm{d} r, \\
m_{t} \in & -\partial I_{[0, \infty)}(m)-h\left(\chi_{t}\right)+\int_{0}^{1} \lambda(x-y) D\left[m, \chi, w_{x x}\right](y, t) \mathrm{d} y,
\end{aligned}
$$

for unknown functions $u, w, \theta, m, \chi$, with initial conditions

$$
\left.\begin{array}{rl}
w(x, 0) & =u(x, 0)=0, \\
m(x, 0) & =m^{0}(x)=0, \\
\theta(x, 0) & =\theta^{0}(x), \\
\chi(x, 0) & =\chi^{0}(x),
\end{array}\right\}
$$

and boundary conditions

$$
\left.\begin{array}{rl}
w(0, t) & =u(0, t)=w(1, t)=u(1, t)=0 \\
\theta_{x}(0, t) & =\theta_{x}(1, t)=0
\end{array}\right\}
$$

The zero initial conditions for $w$ and $m$ are motivated by the fact that it is difficult to determine the initial degree of fatigue for a material with unknown loading history, and the most transparent hypothesis consists in assuming that no deformation (and therefore no fatigue) has taken place prior to the time $t=0$.

The data are required to fulfill the following hypotheses:

\section{Hypothesis 4.1 .}

(i) $P$ is a Prandtl-Ishlinskii operator (2.5) with a measurable distribution function $\varphi:[0, \infty) \times[0,1] \times$ $(0, \infty) \rightarrow[0, \infty)$, locally Lipschitz continuous in the first two variables, and there exist $\tilde{\varphi}, \varphi^{*} \in L^{1}(0, \infty)$ such that $\varphi(m, \chi, r) \leq \tilde{\varphi}(r), 0 \leq-\varphi_{m}(m, \chi, r) \leq \varphi^{*}(r),-\varphi^{*}(r) \leq \varphi_{\chi}(m, \chi, r) \leq \varphi^{*}(r),\left|\varphi_{m m}(m, \chi, r)\right| \leq$ $\varphi^{*}(r)$ a.e., $\left|\varphi_{\chi m}(m, \chi, r)\right| \leq \varphi^{*}(r)$ a.e., $\left|\varphi_{\chi \chi}(m, \chi, r)\right| \leq \varphi^{*}(r)$, with $M:=\int_{0}^{\infty} r \tilde{\varphi}(r) \mathrm{d} r<\infty$ and also $\int_{0}^{\infty}\left(1+r^{2}\right) \varphi^{*}(r) \mathrm{d} r<2 L$, being $L$ the latent heat of the process.

(ii) $B, \nu, \beta, \theta_{\text {ref }}, \rho, \alpha, c, \kappa, L, \gamma$ are given positive constants.

(iii) $\lambda: \mathbb{R} \rightarrow[0, \infty)$ is a $C^{1}$ function with compact support, and we set $\Lambda:=\max \left\{\lambda(x)+\left|\lambda^{\prime}(x)\right|, x \in \mathbb{R}\right\}$.

(iv) $f \in L^{2}\left(\Omega_{T}\right)$ is a given function for some fixed $T>0$, such that $f_{x}, f_{t t}, f_{x t} \in L^{2}\left(\Omega_{T}\right)$.

(v) $\theta^{0} \in L^{\infty}(0,1)$ and $\chi^{0} \in W^{1,2}(0,1)$ are such that $\theta^{0} \geq \theta_{*}>0, \theta_{x}^{0} \in L^{2}(0,1), \chi^{0}(x) \in[0,1]$ for all $x \in[0,1]$.

(vi) $h: \mathbb{R} \rightarrow[0, \infty)$ is a nondecreasing Lipschitz continuous function such that $0 \leq h^{\prime}(z) \leq a$ a.e. and $h(z) \leq b z^{2}$ for $z \in \mathbb{R}$, where $a, b$ are positive constants; in particular $b$ is such that $b L \leq \gamma / 2$, where $L$ is as in (i) and $\gamma$ is the relaxation coefficient from (4.4).

(vii) $g:[0, \infty) \times \Omega_{T} \rightarrow \mathbb{R}$ is a Carathéodory function such that $g_{0}(x, t):=g(0, x, t) \geq 0, g_{0} \in L^{2}\left(\Omega_{T}\right)$, and $\left|g_{\theta}(\theta, x, t)\right| \leq g_{1}$ a.e. with $g_{1}$ a constant. 
The assumptions required on the function $h$ are needed for the proof of the thermodynamic consistency of the model, for more details see [4]; moreover the assumption of boundedness of the derivative of $h$ in (vi) is used to show the positivity of the temperature.

The assumption that $\varphi(m, \chi, r)$ decreases with increasing fatigue $m$ corresponds to the observation that the stiffness of the material decreases with increasing fatigue. Moreover, the assumptions on $\varphi_{\chi}$ allow us to conclude that the last summand in (3.9) is bounded by the latent heat, which is physically reasonable. Also the assumption that $g_{0}(x, t) \geq 0$ makes sense. Note that $g$ is the heat source density, so that at zero temperature, we cannot remove heat from the system.

We now check that regular solutions to (4.1)-(4.7) satisfy (3.7) with $\varepsilon=w_{x x}$ and $\sigma$ given by (3.1). Indeed, we have by (3.4), (4.3), and (4.4)

$$
\mathcal{S}(\theta, \chi, \varepsilon)_{t}+\left(\frac{q}{\theta}\right)_{x}-\frac{g}{\theta}=\frac{\kappa \theta_{x}^{2}}{\theta^{2}}+\frac{\nu \varepsilon_{t}^{2}}{\theta}+\frac{1}{\theta}\left(D[m, \chi, \varepsilon](t)-\frac{1}{2} m_{t} \int_{0}^{\infty} \varphi_{m}(m, \chi, r) \mathfrak{s}_{r}^{2}[\varepsilon](t) \mathrm{d} r+\gamma \chi_{t}^{2}\right) .
$$

By Hypothesis 4.1 (i) and (4.5)

$$
D[m, \chi, \varepsilon](t)-\frac{1}{2} m_{t} \int_{0}^{\infty} \varphi_{m}(m, \chi, r) \mathfrak{s}_{r}^{2}[\varepsilon](t) \mathrm{d} r+\gamma \chi_{t}^{2} \geq \gamma \chi_{t}^{2}-\frac{1}{2} \cdot 2 L h\left(\chi_{t}\right),
$$

hence, by Hypothesis 4.1 (vi)

$$
\mathcal{S}(\theta, \chi, \varepsilon)_{t}+\left(\frac{q}{\theta}\right)_{x}-\frac{g}{\theta} \geq \frac{\kappa \theta_{x}^{2}}{\theta^{2}}+\frac{\nu \varepsilon_{t}^{2}}{\theta}+\frac{\gamma \chi_{t}^{2}}{2 \theta} \geq 0
$$

provided we check that the absolute temperature $\theta$ stays positive. In Subsection 6.1, we will find a positive lower bound for the discrete approximations of the temperature, which is independent of the discretization parameter, and therefore it is preserved in the limit and it implies the positivity of the temperature.

The main result of this paper reads as follows.

Theorem 4.2. Let Hypothesis 4.1 hold. Then there exists a unique solution to the system (4.1)-(4.7) in $\Omega_{T}$ such that $\theta(x, t)>0$ for all $(x, t) \in \Omega_{T}$, and with the regularity

- $w_{x x x t}, w_{x x t t}, \theta_{t}, \theta_{x x}, u_{t t}, u_{x x t} \in L^{2}\left(\Omega_{T}\right)$,

- $\theta, m_{t}, \chi_{t} \in L^{\infty}\left(\Omega_{T}\right)$.

The proof of the existence result is carried out in three steps: approximation, a priori estimates and passage to the limit. We will follow the lines of [13], trying to focus on the main differences, due to the presence of the new terms.

\section{Approximation}

From now on, the values of all physical constants are set to 1 for simplicity, with the exception of $\gamma, L, \theta_{\text {ref }}$ in order to emphasize the role of the phase transition.

Let us choose an integer $n \in \mathbb{N}$ and consider an equidistant partition of the interval $[0,1]$. Let us take the space discrete approximations of (4.1)-(4.5) for $k=1, \ldots, n-1$ :

$$
\begin{aligned}
\dot{u}_{k} & =\varepsilon_{k}+P\left[m_{k}, \chi_{k}, \varepsilon_{k}\right]+\dot{\varepsilon}_{k}-\theta_{k}+\theta_{\text {ref }}, \\
\dot{w}_{k}-\dot{\varepsilon}_{k} & =-n^{2}\left(u_{k+1}-2 u_{k}+u_{k-1}\right)+f_{k}, \\
\varepsilon_{k} & =n^{2}\left(w_{k+1}-2 w_{k}+w_{k-1}\right), \\
\dot{\theta}_{k} & =n^{2}\left(\theta_{k+1}-2 \theta_{k}+\theta_{k-1}\right)+\dot{m}_{k} \mathcal{K}_{k}+\dot{\chi}_{k}\left(\mathcal{M}_{k}-L\right)+D_{k}+\dot{\varepsilon}_{k}^{2}-\theta_{k} \dot{\varepsilon}_{k}+g_{k}\left(\theta_{k}, t\right), \\
\gamma \chi_{k} & =\mathfrak{s}_{[0,1]}\left[\chi_{k}^{0}, A_{k}\right], \quad A_{k}(t)=\int_{0}^{t}\left(\frac{L}{\theta_{\text {ref }}}\left(\theta_{k}-\theta_{\text {ref }}\right)+\mathcal{M}_{k}\right)(\tau) \mathrm{d} \tau,
\end{aligned}
$$




$$
m_{k}=\mathfrak{s}_{[0, \infty)}\left[0, S_{k}\right], \quad S_{k}(t)=\int_{0}^{t}\left(-h\left(\dot{\chi}_{k}\right)+\mathcal{D}_{k}^{*}\right)(\tau) \mathrm{d} \tau
$$

where

$$
\begin{aligned}
& \mathcal{K}_{k}(t)=-\frac{1}{2} \int_{0}^{\infty} \varphi_{m}\left(m_{k}(t), \chi_{k}(t), r\right) \mathfrak{s}_{r}^{2}\left[\varepsilon_{k}\right](t) \mathrm{d} r, \\
& \mathcal{M}_{k}(t)=-\frac{1}{2} \int_{0}^{\infty} \varphi_{\chi}\left(m_{k}(t), \chi_{k}(t), r\right) \mathfrak{s}_{r}^{2}\left[\varepsilon_{k}\right](t) \mathrm{d} r, \\
& D_{k}(t)=\int_{0}^{\infty} \varphi\left(m_{k}(t), \chi_{k}(t), r\right) \mathfrak{s}_{r}\left[\varepsilon_{k}\right](t)\left(\varepsilon_{k}-\mathfrak{s}_{r}\left[\varepsilon_{k}\right]\right)_{t}(t) \mathrm{d} r \geq 0, \\
& \mathcal{D}_{k}^{*}(t)=\frac{1}{n} \sum_{j=1}^{n-1} \lambda_{k-j} D_{j}(t) \geq 0, \\
& \lambda_{i}=\lambda(i / n), \\
& f_{k}(t)=n \int_{(k-1) / n}^{k / n} f(x, t) \mathrm{d} x, \\
& g_{k}(\theta, t)=\left\{\begin{array}{cc}
n \int_{(k-1) / n}^{k / n} & \text { for } \theta<0 . \\
g_{k}(0, t) & \text { for } \theta, t) \mathrm{d} x \text { for } \theta \geq 0,
\end{array}\right.
\end{aligned}
$$

Let us notice that, by Hypothesis $4.1(\mathrm{i})$, we have $\mathcal{K}_{k}(t) \in[0, L],\left|\mathcal{M}_{k}(t)\right| \leq L$ for a.e. $t \in[0, T]$. We prescribe initial conditions for $k=1, \ldots, n-1$

$$
\left.\begin{array}{l}
w_{k}(0)=u_{k}(0)=0, \\
\theta_{k}(0)=\theta_{k}^{0}:=\theta^{0}(k / n), \\
m_{k}(0)=0, \\
\chi_{k}(0)=\chi_{k}^{0}:=n \int_{(k-1) / n}^{k / n} \chi^{0}(x) \mathrm{d} x,
\end{array}\right\}
$$

and "boundary conditions"

$$
\left.\begin{array}{l}
w_{0}=w_{n}=u_{0}=u_{n}=0 \\
\theta_{0}=\theta_{1}, \theta_{n}=\theta_{n-1}
\end{array}\right\}
$$

This turns to be a system of ODEs for the unknowns $u_{k}, w_{k}, \theta_{k}, \chi_{k}, m_{k}$, with a locally Lipschitz continuous and locally bounded right hand side. This follows from the properties of the stop operator, namely Proposition 2.2 (ii). Therefore, from the standard theory of ODEs, it is possible to deduce the existence and uniqueness of a local solution in an interval $\left[0, T_{n}\right]$ and the solution belongs to $W^{1, \infty}\left(0, T_{n}\right)$. We will show at the end of Subsection 6.2 that the solution exists globally, and actually $T_{n}=T$.

\section{A PRIORI ESTIMATES}

This section is divided into 4 subsections:

- Positivity of the temperature: it is important for the thermodynamical consistency of the model;

- Discrete energy inequality: it constitutes the basic a priori estimate giving the first minimal regularity (which is however not enough to pass to the limit); 
- Discrete Dafermos estimates: it provides additional regularity for the temperature, necessary to deduce further a priori estimates;

- Higher order estimates: this subsection provides the necessary estimates to be able to proceed with the passage to the limit.

In the sequel, we will systematically use the "summation by parts formula"

$$
\sum_{k=1}^{n-1} \xi_{k}\left(\eta_{k+1}-2 \eta_{k}+\eta_{k-1}\right)+\sum_{k=1}^{n}\left(\xi_{k}-\xi_{k-1}\right)\left(\eta_{k}-\eta_{k-1}\right)=\xi_{n}\left(\eta_{n}-\eta_{n-1}\right)-\xi_{0}\left(\eta_{1}-\eta_{0}\right)
$$

for all vectors $\left(\xi_{0}, \ldots, \xi_{n}\right),\left(\eta_{0}, \ldots, \eta_{n}\right)$.

\subsection{Positivity of the temperature}

First of all it is important to prove that all $\theta_{k}$ remain positive in the whole range of existence. We test (5.4) by $-\theta_{k}^{-}$, where $\theta_{k}^{-}$is the negative part of $\theta_{k}$, getting on one hand

$$
-\frac{1}{n} \sum_{k=1}^{n-1} \dot{\theta}_{k} \theta_{k}^{-}=\frac{1}{2 n} \frac{\mathrm{d}}{\mathrm{d} t} \sum_{k=1}^{n-1}\left(\theta_{k}^{-}\right)^{2}
$$

Moreover, by (6.1) we also deduce

$$
-n \sum_{k=1}^{n-1}\left(\theta_{k+1}-2 \theta_{k}+\theta_{k-1}\right) \theta_{k}^{-}=n \sum_{k=1}^{n}\left(\theta_{k}-\theta_{k-1}\right)\left(\theta_{k}^{-}-\theta_{k-1}^{-}\right) \leq-n \sum_{n=1}^{n}\left(\theta_{k}^{-}-\theta_{k-1}^{-}\right)^{2} \leq 0 .
$$

On the other hand, having $D_{k}(t) \geq 0$ and $g_{k}(\theta, t) \geq 0$ for $\theta \leq 0$ by Hypothesis 4.1 (vii), it follows that

$$
-\left(D_{k}(t)+\dot{\varepsilon}_{k}^{2}(t)+g_{k}\left(\theta_{k}, t\right)\right) \theta_{k}^{-} \leq 0 .
$$

By Hypothesis 4.1 (i) for the phase term we have

$$
\left.\begin{array}{ll}
\dot{\chi}_{k}(t) \theta_{k}^{-}(t)=0 & \text { if } \dot{\chi}_{k}(t)=0, \\
\dot{\chi}_{k}(t) \theta_{k}^{-}(t)=\frac{L}{\theta_{\text {ref }}}\left(\theta_{k}(t)-\theta_{\text {ref }}\right) \theta_{k}^{-}(t)+\frac{\mathcal{M}_{k}}{\gamma} \theta_{k}^{-}(t) \leq 0 & \text { otherwise, }
\end{array}\right\}
$$

therefore

$$
-\left(\mathcal{M}_{k}-L\right) \dot{\chi}_{k}(t) \theta_{k}^{-}(t) \leq 0 .
$$

Finally, (6.2) and Hypothesis 4.1 (vi) allow us to show that if $\dot{m}_{k}(t) \neq 0$

$$
-\dot{m}_{k}(t) \mathcal{K}_{k}(t) \theta_{k}^{-}(t)=\left(h\left(\dot{\chi}_{k}(t)\right)-\mathcal{D}_{k}^{*}(t)\right) \theta_{k}^{-}(t) \mathcal{K}_{k}(t) \leq h\left(\dot{\chi}_{k}(t)\right) \theta_{k}^{-}(t) \mathcal{K}_{k}(t) \leq 0 .
$$

Putting together the previous estimates and setting

$$
K_{n}:=\max \left\{\left|\dot{\varepsilon}_{k}(t)\right|: k=1, \ldots, n-1, \quad t \in\left[0, T_{n}\right]\right\},
$$

we come to

$$
\frac{\mathrm{d}}{\mathrm{d} t} \frac{1}{2 n} \sum_{k=1}^{n-1}\left(\theta_{k}^{-}\right)^{2} \leq \frac{1}{n} \sum_{k=1}^{n-1}\left(\theta_{k}^{-}\right)^{2}\left|\dot{\varepsilon}_{k}\right| \leq \frac{K_{n}}{n} \sum_{k=1}^{n-1}\left(\theta_{k}^{-}\right)^{2} .
$$

Gronwall's argument now allows us to obtain $\theta_{k}^{-}(t)=0$ for all $k$ and $t \in\left[0, T_{n}\right]$. 
At this point we prove that in addition $\theta_{k}(t)$ are bounded away from 0 for all $k$ and all $t \in\left[0, T_{n}\right]$. More precisely, if $p$ is the solution of the differential equation

$$
\dot{p}+\psi(p)=0, \quad p(0)=\theta_{*},
$$

with $\theta_{*}>0$ from Hypothesis $4.1(\mathrm{v})$, and where $\psi$ is a suitable function of polynomial kind we will choose later, then we are able to show that $\theta_{k}(t) \geq p(t)>0$ for all $k$ and all $t \in\left[0, T_{n}\right]$.

First of all, if $\dot{\chi}_{k} \neq 0$ then

$$
-\gamma \dot{\chi}_{k}=-\frac{L}{\theta_{\text {ref }}}\left(\theta_{k}-\theta_{\text {ref }}\right)-\mathcal{M}_{k} \geq-\frac{L \theta_{k}}{\theta_{\text {ref }}}
$$

so that

$$
\dot{\chi}_{k}\left(\mathcal{M}_{k}-L\right) \geq \frac{L \theta_{k}}{\theta_{\text {ref }}}\left(\mathcal{M}_{k}-L\right) \geq-\frac{2 L^{2}}{\theta_{\text {ref }}} \theta_{k}
$$

On the other hand

$$
\dot{m}_{k} \geq-h\left(\dot{\chi}_{k}\right) \geq-a \dot{\chi}_{k} \geq-\frac{L a \theta_{k}}{\theta_{\text {ref }} \gamma}
$$

from which we deduce

$$
\dot{m}_{k} \mathcal{K}_{k} \geq-\frac{a L^{2}}{\theta_{\text {ref }} \gamma} \theta_{k}
$$

Moreover, by Hypothesis 4.1 (vii)

$$
g_{k} \geq n \int_{(k-1) / n}^{k / n}\left(g\left(\theta_{k}, x, t\right)-g(0, x, t)\right) \mathrm{d} x \geq n \int_{(k-1) / n}^{k / n}\left(-g_{1}\right) \theta_{k} \mathrm{~d} x=-g_{1} \theta_{k} .
$$

We additionally have

$$
\dot{\varepsilon}_{k}^{2}-\theta_{k} \dot{\varepsilon}_{k} \geq-\frac{1}{4} \theta_{k}^{2}
$$

Summing up all together we deduce from (5.4)

$$
\dot{\theta}_{k}-n^{2}\left(\theta_{k+1}-2 \theta_{k}+\theta_{k-1}\right) \geq-\psi\left(\theta_{k}\right)
$$

with

$$
\psi(z):=\frac{1}{4} z^{2}+\left[\frac{L^{2}}{\theta_{\mathrm{ref} \gamma}}(2+a)+g_{1}\right] z .
$$

On the other hand we have from (6.3) that

$$
p(t)=\frac{\mu \theta_{*} e^{-\mu t}}{\delta \theta_{*}\left(1-e^{-\mu t}\right)+\mu} \quad \text { with } \quad \delta=\frac{1}{4}, \quad \mu=\frac{L^{2}}{\theta_{\text {ref }} \gamma}(2+a)+g_{1} .
$$

At this point we compare $\theta_{k}$ and $p$. We have

$$
\left(\dot{p}-\dot{\theta}_{k}\right)-n^{2}\left(\left(p-\theta_{k+1}\right)-2\left(p-\theta_{k}\right)+\left(p-\theta_{k-1}\right)\right)+\psi(p)-\psi\left(\theta_{k}\right) \leq 0,
$$

so testing (6.7) by $\left(p-\theta_{k}\right)^{+}$and using (6.1), we deduce

$$
\frac{1}{2} \frac{\mathrm{d}}{\mathrm{d} t} \sum_{k=1}^{n-1}\left(\left(p-\theta_{k}\right)^{+}\right)^{2}+n^{2} \sum_{k=1}^{n}\left(\left(p-\theta_{k}\right)^{+}-\left(p-\theta_{k-1}\right)^{+}\right)^{2}+\left(\psi(p)-\psi\left(\theta_{k}\right)\right)\left(p-\theta_{k}\right)^{+} \leq 0 .
$$


But $\psi$ is nondecreasing for positive arguments, so

$$
\sum_{k=1}^{n-1}\left(\left(p-\theta_{k}\right)^{+}\right)^{2}(t) \leq \sum_{k=1}^{n-1}\left(\left(p-\theta_{k}\right)^{+}\right)^{2}(0)=0,
$$

which yields the desired result.

\subsection{Discrete energy estimate}

We obtain the usual energy estimate by testing (5.1) by $\dot{\varepsilon}_{k}$, differentiating (5.2) in time and testing it by $\dot{w}_{k}$, summing then the result over $k=1, \ldots, n-1$. From (5.3), with a repeated use of (5.8) and (6.1), we obtain

$$
\begin{aligned}
& \frac{\mathrm{d}}{\mathrm{d} t}\left(\frac{1}{2 n} \sum_{k=1}^{n-1} \dot{w}_{k}^{2}+\frac{n}{2} \sum_{k=1}^{n}\left(\dot{w}_{k}-\dot{w}_{k-1}\right)^{2}\right) \\
& \quad+\frac{1}{n} \sum_{k=1}^{n-1} \dot{\varepsilon}_{k}\left(\varepsilon_{k}+P\left[m_{k}, \chi_{k}, \varepsilon_{k}\right]+\dot{\varepsilon}_{k}-\theta_{k}+\theta_{\text {ref }}\right)=\frac{1}{n} \sum_{k=1}^{n-1} \dot{f}_{k} \dot{w}_{k} .
\end{aligned}
$$

Adding (6.9) to (5.4) allows us to obtain, by virtue of (2.8),

$$
\begin{gathered}
\frac{\mathrm{d}}{\mathrm{d} t}\left(\frac{1}{n} \sum_{k=1}^{n-1}\left(\frac{1}{2} \varepsilon_{k}^{2}+V\left[m_{k}, \chi_{k}, \varepsilon_{k}\right]+\theta_{\mathrm{ref}} \varepsilon_{k}+\frac{1}{2} \dot{w}_{k}^{2}+\theta_{k}+\chi_{k}\right)\right. \\
\left.+\frac{n}{2} \sum_{k=1}^{n}\left(\dot{w}_{k}-\dot{w}_{k-1}\right)^{2}\right)=\frac{1}{n} \sum_{k=1}^{n-1}\left(\dot{f}_{k} \dot{w}_{k}+g_{k}\right) .
\end{gathered}
$$

Estimating the right hand side of (6.10) using a discrete version of Hölder inequality, and using Hypothesis 4.1 (vii) together with Gronwall's lemma, we conclude

$$
\frac{1}{n} \sum_{k=1}^{n-1}\left(\dot{w}_{k}^{2}+\varepsilon_{k}^{2}+\theta_{k}\right)(t)+n \sum_{k=1}^{n}\left(\dot{w}_{k}-\dot{w}_{k-1}\right)^{2}(t) \leq C .
$$

In particular the approximate solutions exist globally and $T_{n}=T$.

\subsection{Discrete Dafermos estimate}

We test (5.4) by $\theta_{k}^{-1 / 3}$ and obtain for all $t \in[0, T]$, after summing up from $k=1, \ldots, n-1$

$$
\begin{aligned}
0= & \sum_{k=1}^{n-1}\left(-\dot{\theta}_{k} \theta_{k}^{-1 / 3}\right)+n^{2} \sum_{k=1}^{n-1}\left(\theta_{k-1}-2 \theta_{k}+\theta_{k-1}\right) \theta_{k}^{-1 / 3} \\
& +\sum_{k=1}^{n-1} \dot{m}_{k} \mathcal{K}_{k} \theta_{k}^{-1 / 3}+\sum_{k=1}^{n-1} \mathcal{D}_{k} \theta_{k}^{-1 / 3} \\
& +\sum_{k=1}^{n-1} \dot{\varepsilon}_{k}^{2} \theta_{k}^{-1 / 3}-\sum_{k=1}^{n-1} \theta_{k} \dot{\varepsilon}_{k} \theta_{k}^{-1 / 3} \\
& +\sum_{k=1}^{n-1} \dot{\chi}_{k}\left(M_{k}-L\right) \theta_{k}^{-1 / 3}+\sum_{k=1}^{n-1} g_{k} \theta_{k}^{-1 / 3} \\
=: & T_{1}+T_{2}+T_{3}+T_{4}+T_{5}+T_{6}+T_{7}+T_{8} .
\end{aligned}
$$


Observe that $T_{4} \geq 0$, hence

$$
T_{1}+T_{2}+T_{3}+T_{5}+T_{6}+T_{7}+T_{8} \leq 0 .
$$

We estimate the remaining terms. Using the summation by parts formula (6.1) and the elementary inequality

$$
-\left(\theta_{k}-\theta_{k-1}\right)\left(\theta_{k}^{-1 / 3}-\theta_{k-1}^{-1 / 3}\right) \geq 3\left(\theta_{k}^{1 / 3}-\theta_{k-1}^{1 / 3}\right)^{2},
$$

we deduce

$$
\begin{aligned}
T_{2} & :=n^{2} \sum_{k=1}^{n-1}\left(\theta_{k-1}-2 \theta_{k}+\theta_{k-1}\right) \theta_{k}^{-1 / 3} \\
& =-n^{2} \sum_{k=1}^{n}\left(\theta_{k}^{-1 / 3}-\theta_{k-1}^{-1 / 3}\right)\left(\theta_{k}-\theta_{k-1}\right)+n^{2}\left[\theta_{n}^{-1 / 3}\left(\theta_{n}-\theta_{n-1}\right)-\theta_{0}^{-1 / 3}\left(\theta_{1}-\theta_{0}\right)\right] \\
& \geq 3 n^{2} \sum_{k=1}^{n}\left(\theta_{k}^{1 / 3}-\theta_{k-1}^{1 / 3}\right)^{2}
\end{aligned}
$$

where the term $n^{2}\left[\theta_{n}^{-1 / 3}\left(\theta_{n}-\theta_{n-1}\right)-\theta_{0}^{-1 / 3}\left(\theta_{1}-\theta_{0}\right)\right]$ vanishes due to (5.8).

Concerning the term $T_{3}$, we observe that $T_{3} \geq 0$ if $\dot{m}_{k} \geq 0$ while in the case $\dot{m}_{k}<0$, by (6.5)

$$
T_{3}:=\sum_{k=1}^{n-1} \dot{m}_{k} \mathcal{K}_{k} \theta_{k}^{-1 / 3} \geq-\frac{a L^{2}}{\gamma \theta_{\text {ref }}} \sum_{k=1}^{n-1} \theta_{k}^{2 / 3} .
$$

The same happens for the term $T_{7}$ noticing that, by (6.4)

$$
\gamma \dot{\chi}_{k}=\frac{L}{\theta_{\text {ref }}} \theta_{k}-L+\mathcal{M}_{k} \leq \frac{L}{\theta_{\text {ref }}} \theta_{k}
$$

therefore

$$
T_{7}:=\sum_{k=1}^{n-1} \dot{\chi}_{k}\left(\mathcal{M}_{k}-L\right) \theta_{k}^{-1 / 3} \geq-\frac{2 L^{2}}{\gamma \theta_{\text {ref }}} \sum_{k=1}^{n-1} \theta_{k}^{2 / 3} .
$$

Concerning the term $T_{8}$, by (6.6) we have

$$
T_{8}:=\sum_{k=1}^{n-1} g_{k} \theta_{k}^{-1 / 3} \geq-g_{1} \sum_{k=1}^{n-1} \theta_{k}^{2 / 3} .
$$

By estimating the terms $T_{1}$ and $T_{6}$ from above, dividing the final inequality by $n$ and integrating in time we finally get

$$
\begin{aligned}
& \int_{0}^{t}\left(3 n \sum_{k=1}^{n}\left(\theta_{k}^{1 / 3}-\theta_{k-1}^{1 / 3}\right)^{2}+\frac{1}{n} \sum_{k=1}^{n-1} \dot{\varepsilon}_{k}^{2} \theta_{k}^{-1 / 3}\right) \mathrm{d} \tau \\
\leq & \int_{0}^{t} \frac{1}{n} \sum_{k=1}^{n-1}\left|\dot{\varepsilon}_{k}\right| \theta_{k}^{2 / 3} \mathrm{~d} \tau+\frac{3}{2 n} \sum_{k=1}^{n-1} \theta_{k}^{2 / 3}(t)+C \int_{0}^{t} \frac{1}{n} \sum_{k=1}^{n-1} \theta_{k}^{2 / 3} \mathrm{~d} \tau,
\end{aligned}
$$

where the constant $C$ depends on $L, \gamma, M, \theta_{\text {ref }}, g_{1}$ but is independent of $n$ and where also the term $T_{3}+T_{7}+T_{8}$ integrated in time, due to the minus sign, goes to the right hand side of the estimate. 
The last two terms on the right hand side are bounded by virtue of (6.11). The conclusion now comes in a similar way as in the paper [13], by means of discrete versions of Hölder and Gagliardo-Nirenberg inequalities, in order to estimate the first term in the right hand side of (6.13). This gives

$$
\int_{0}^{t} \frac{1}{n} \sum_{k=1}^{n-1} \theta_{k}^{8 / 3}(\tau) \mathrm{d} \tau \leq C,
$$

and

$$
\int_{0}^{t} \frac{1}{n} \sum_{k=1}^{n-1} \dot{\varepsilon}_{k}^{2}(\tau) \mathrm{d} \tau \leq C .
$$

\subsection{Higher order discrete estimates}

Before continuing, we need to extend the validity of (5.1), (5.5), (5.6) to $k=0$ and $k=n$. For this purpose for $k=0$ we solve the system of these three equations in the unknowns $\varepsilon_{0}, \chi_{0}, m_{0}$, and for $k=n$ in the unknowns $\varepsilon_{n}, \chi_{n}, m_{n}$. By Proposition 2.2 both systems admit Lipschitz continuous right hand sides, which implies that $\varepsilon_{0}, \chi_{0}, m_{0}$ and $\varepsilon_{n}, \chi_{n}, m_{n}$ are determined uniquely. In this way (5.1) makes sense for all $k=0, \ldots, n$; thus we can take space increments as follows

$$
\dot{u}_{k}-\dot{u}_{k-1}=\varepsilon_{k}-\varepsilon_{k-1}+P\left[m_{k}, \chi_{k}, \varepsilon_{k}\right]-P\left[m_{k-1}, \chi_{k-1}, \varepsilon_{k-1}\right]+\dot{\varepsilon}_{k}-\dot{\varepsilon}_{k-1}-\theta_{k}+\theta_{k-1},
$$

for all $k=1, \ldots, n$. The summation by parts formula (6.1) gives

$$
n \sum_{k=1}^{n}\left(\dot{u}_{k}-\dot{u}_{k-1}\right)^{2}=-n \sum_{k=1}^{n-1} \dot{u}_{k}\left(\dot{u}_{k+1}-2 \dot{u}_{k}+\dot{u}_{k-1}\right),
$$

hence, by (5.1)-(5.2) and (6.16),

$$
\begin{aligned}
& n \sum_{k=1}^{n}\left(\varepsilon_{k}-\varepsilon_{k-1}+P\left[m_{k}, \chi_{k}, \varepsilon_{k}\right]-P\left[m_{k-1}, \chi_{k-1}, \varepsilon_{k-1}\right]+\dot{\varepsilon}_{k}-\dot{\varepsilon}_{k-1}-\theta_{k}+\theta_{k-1}\right)^{2} \\
& \quad=\frac{1}{n} \sum_{k=1}^{n-1}\left(\ddot{w}_{k}-\ddot{\varepsilon}_{k}-\dot{f}_{k}\right)\left(\varepsilon_{k}+P\left[m_{k}, \chi_{k}, \varepsilon_{k}\right]+\dot{\varepsilon}_{k}-\theta_{k}+\theta_{\text {ref }}\right) .
\end{aligned}
$$

Using the implication $(a+b)^{2}=X \Rightarrow a^{2} / 2 \leq 3 b^{2}+X, \forall a, b, X \in \mathbb{R}$, we estimate the first term in the left hand side of (6.17) as follows

$$
\begin{aligned}
n \sum_{k=1}^{n}\left(\left(\varepsilon_{k}-\varepsilon_{k-1}\right)^{2}+\left(\dot{\varepsilon}_{k}-\dot{\varepsilon}_{k-1}\right)^{2}\right)+\frac{\mathrm{d}}{\mathrm{d} t} & {\left[n \sum_{k=1}^{n}\left(\varepsilon_{k}-\varepsilon_{k-1}\right)^{2}\right] } \\
=n \sum_{k=1}^{n}\left(\left(\varepsilon_{k}-\varepsilon_{k-1}\right)+\left(\dot{\varepsilon}_{k}-\dot{\varepsilon}_{k-1}\right)\right)^{2} \leq & 6 n \sum_{k=1}^{n}\left(\left(P\left[m_{k}, \chi_{k}, \varepsilon_{k}\right]-P\left[m_{k-1}, \chi_{k-1}, \varepsilon_{k-1}\right]\right)^{2}+\left(\theta_{k}-\theta_{k-1}\right)^{2}\right) \\
+ & \frac{2}{n} \sum_{k=1}^{n-1}\left(\ddot{w}_{k}-\ddot{\varepsilon}_{k}-\dot{f}_{k}\right)\left(\varepsilon_{k}+P\left[m_{k}, \chi_{k}, \varepsilon_{k}\right]+\dot{\varepsilon}_{k}-\theta_{k}+\theta_{\text {ref }}\right) .
\end{aligned}
$$

On the other hand we have

$$
\frac{1}{n} \sum_{k=1}^{n-1}\left(\ddot{w}_{k}-\ddot{\varepsilon}_{k}-\dot{f}_{k}\right)\left(\varepsilon_{k}+P\left[m_{k}, \chi_{k}, \varepsilon_{k}\right]+\dot{\varepsilon}_{k}-\theta_{k}+\theta_{\text {ref }}\right)
$$




$$
\begin{aligned}
= & \frac{1}{n} \sum_{k=1}^{n-1}\left(\ddot{w}_{k}-\ddot{\varepsilon}_{k}\right) \dot{\varepsilon}_{k}+\frac{1}{n} \sum_{k=1}^{n-1}\left(\ddot{w}_{k}-\ddot{\varepsilon}_{k}\right)\left(\varepsilon_{k}+P\left[m_{k}, \chi_{k}, \varepsilon_{k}\right]-\theta_{k}+\theta_{\text {ref }}\right) \\
& -\frac{1}{n} \sum_{k=1}^{n-1} \dot{f}_{k}\left(\varepsilon_{k}+P\left[m_{k}, \chi_{k}, \varepsilon_{k}\right]+\dot{\varepsilon}_{k}-\theta_{k}+\theta_{\text {ref }}\right) .
\end{aligned}
$$

In particular, using (5.3) we deduce

$$
\begin{aligned}
\frac{1}{n} \sum_{k=1}^{n-1}\left(\ddot{w}_{k}-\ddot{\varepsilon}_{k}\right) \dot{\varepsilon}_{k} & =\sum_{k=1}^{n-1} \ddot{w}_{k} n\left(\dot{w}_{k+1}-2 \dot{w}_{k}+\dot{w}_{k-1}\right)-\frac{1}{2 n} \frac{\mathrm{d}}{\mathrm{d} t}\left[\sum_{k=1}^{n-1} \dot{\varepsilon}_{k}^{2}\right] \\
& =-\frac{\mathrm{d}}{\mathrm{d} t}\left(\frac{n}{2} \sum_{k=1}^{n}\left(\dot{w}_{k}-\dot{w}_{k-1}\right)^{2}+\frac{1}{2 n} \sum_{k=1}^{n-1} \dot{\varepsilon}_{k}^{2}\right),
\end{aligned}
$$

where we exploited integration by parts and the boundary conditions for $w_{k}$. Putting together all the estimates we finally obtain

$$
\begin{aligned}
& \frac{\mathrm{d}}{\mathrm{d} t}\left(\frac{n}{2} \sum_{k=1}^{n}\left(\dot{w}_{k}-\dot{w}_{k-1}\right)^{2}+\frac{1}{2 n} \sum_{k=1}^{n-1} \dot{\varepsilon}_{k}^{2}+n \sum_{k=1}^{n}\left(\varepsilon_{k}-\varepsilon_{k-1}\right)^{2}\right) \\
& +n \sum_{k=1}^{n}\left(\left(\varepsilon_{k}-\varepsilon_{k-1}\right)^{2}+\left(\dot{\varepsilon}_{k}-\dot{\varepsilon}_{k-1}\right)^{2}\right) \\
& \leq C\left(n \sum_{k=1}^{n}\left(\left(P\left[m_{k}, \chi_{k}, \varepsilon_{k}\right]-P\left[m_{k-1}, \chi_{k-1}, \varepsilon_{k-1}\right]\right)^{2}+\left(\theta_{k}-\theta_{k-1}\right)^{2}\right)\right. \\
& +\frac{1}{n} \sum_{k=1}^{n-1}\left|\dot{f}_{k}\right|\left|\varepsilon_{k}+P\left[m_{k}, \chi_{k}, \varepsilon_{k}\right]+\dot{\varepsilon}_{k}-\theta_{k}+\theta_{\mathrm{ref}}\right| \\
& +\frac{\mathrm{d}}{\mathrm{d} t}\left(\frac{1}{n} \sum_{k=1}^{n-1}\left(\dot{w}_{k}-\dot{\varepsilon}_{k}\right)\left(\varepsilon_{k}+P\left[m_{k}, \chi_{k}, \varepsilon_{k}\right]-\theta_{k}+\theta_{\mathrm{ref}}\right)\right) \\
& \left.+\frac{1}{n} \sum_{k=1}^{n-1}\left|\dot{w}_{k}-\dot{\varepsilon}_{k} \| \dot{\varepsilon}_{k}+P\left[m_{k}, \chi_{k}, \varepsilon_{k}\right]_{t}-\dot{\theta}_{k}\right|\right)=: H_{1}+H_{2}+H_{3}+H_{4} .
\end{aligned}
$$

Let us deal first with the last three terms. Note that we have

$$
\begin{aligned}
\left|P\left[m_{k}, \chi_{k}, \varepsilon_{k}\right]_{t}\right| \leq & \left|\dot{m}_{k} \int_{0}^{\infty} \varphi_{m}\left(m_{k}, \chi_{k}, r\right) \mathfrak{s}_{r}\left[\varepsilon_{k}\right] \mathrm{d} r\right| \\
& +\left|\dot{\chi}_{k} \int_{0}^{\infty} \varphi_{\chi}\left(m_{k}, \chi_{k}, r\right) \mathfrak{s}_{r}\left[\varepsilon_{k}\right] \mathrm{d} r\right|+\left|\int_{0}^{\infty} \varphi\left(m_{k}, \chi_{k}, r\right)\left(\mathfrak{s}_{r}\left[\varepsilon_{k}\right]\right)_{t} \mathrm{~d} r\right| .
\end{aligned}
$$

By (2.2) we have $\left|\mathfrak{s}_{r}\left[\varepsilon_{k}\right]\right| \leq r$, and Proposition 2.2 (iv), together with (5.6), (5.5), (2.9), and Hypothesis 4.1 (i), (iii), (vi) yield

$$
\begin{aligned}
& \left|\mathfrak{s}_{r}\left[\varepsilon_{k}\right]_{t}\right| \leq\left|\dot{\varepsilon}_{k}\right|, \\
& \left|\dot{m}_{k}\right| \leq\left|-h\left(\dot{\chi}_{k}\right)+\mathcal{D}_{k}^{*}\right| \leq C\left(\left|\dot{\chi}_{k}\right|+\frac{1}{n} \sum_{k=1}^{n-1}\left|\dot{\varepsilon}_{k}\right|\right) \\
& \left|\dot{\chi}_{k}\right| \leq C\left(1+\theta_{k}\right) \text { a.e. }
\end{aligned}
$$


From Hypothesis 4.1 (i) we thus obtain the bound

$$
\left|P\left[m_{k}, \chi_{k}, \varepsilon_{k}\right]_{t}\right| \leq C\left(1+\theta_{k}+\frac{1}{n} \sum_{k=1}^{n-1}\left|\dot{\varepsilon}_{k}\right|\right),
$$

and this enables us to obtain the following estimates for the last three terms on the right hand side of (6.21):

$$
\begin{aligned}
H_{2}:=\int_{0}^{t} \frac{1}{n} \sum_{k=1}^{n-1}\left|\dot{f}_{k}\right|\left|\varepsilon_{k}+P\left[m_{k}, \chi_{k}, \varepsilon_{k}\right]+\dot{\varepsilon}_{k}-\theta_{k}+\theta_{\mathrm{ref}}\right| \mathrm{d} \tau \leq C, \\
H_{3}:=\frac{1}{n} \sum_{k=1}^{n-1}\left(\dot{w}_{k}-\dot{\varepsilon}_{k}\right)\left(\varepsilon_{k}+P\left[m_{k}, \chi_{k}, \varepsilon_{k}\right]-\theta_{k}+\theta_{\mathrm{ref}}\right) \\
\leq C\left(1+\left(\frac{1}{n} \sum_{k=1}^{n-1} \dot{\varepsilon}_{k}^{2}\right)^{1 / 2}\right)\left(1+\left(\frac{1}{n} \sum_{k=1}^{n-1} \theta_{k}^{2}\right)^{1 / 2}\right) \\
\leq \frac{1}{4 n} \sum_{k=1}^{n-1} \dot{\varepsilon}_{k}^{2}+C\left(1+\frac{1}{n} \int_{0}^{t} \sum_{k=1}^{n-1} \dot{\theta}_{k}^{2} \mathrm{~d} \tau\right)^{1 / 2},
\end{aligned}
$$

where we used Hölder's inequality together with the a priori estimates obtained before (6.11), (6.14), (6.15) and Hypothesis 4.1 (i), (vi), and also

$$
H_{4}:=\int_{0}^{t} \frac{1}{n} \sum_{k=1}^{n-1}\left|\dot{w}_{k}-\dot{\varepsilon}_{k} \| \dot{\varepsilon}_{k}+P\left[m_{k}, \chi_{k}, \varepsilon_{k}\right]_{t}-\dot{\theta}_{k}\right| \mathrm{d} \tau \leq C\left(1+\left(\int_{0}^{t} \frac{1}{n} \sum_{k=1}^{n-1} \dot{\theta}_{k}^{2} \mathrm{~d} \tau\right)^{1 / 2}\right),
$$

where we used in addition also (6.25). Here and in the sequel the constants $C$ are independent of $n$.

It is a bit more complicated to deal with the term $H_{1}$. First of all we have by Proposition 2.2 and Hypothesis $4.1(\mathrm{i})$

$$
\begin{aligned}
\left|P\left[m_{k}, \chi_{k}, \varepsilon_{k}\right](t)-P\left[m_{k-1}, \chi_{k-1}, \varepsilon_{k-1}\right](t)\right| \\
=\left|\int_{0}^{\infty}\left(\varphi\left(m_{k}, \chi_{k}, r\right) \mathfrak{s}_{r}\left[\varepsilon_{k}\right]-\varphi\left(m_{k-1}, \chi_{k-1}, r\right) \mathfrak{s}_{r}\left[\varepsilon_{k-1}\right]\right) \mathrm{d} r\right| \\
=\mid \int_{0}^{\infty}\left(\left(\varphi\left(m_{k}, \chi_{k}, r\right)-\varphi\left(m_{k-1}, \chi_{k}, r\right)\right) \mathfrak{s}_{r}\left[\varepsilon_{k}\right]\right. \\
\left.\quad+\left(\varphi\left(m_{k-1}, \chi_{k}, r\right)-\varphi\left(m_{k-1}, \chi_{k-1}, r\right)\right) \mathfrak{s}_{r}\left[\varepsilon_{k}\right] \mathrm{d} r\right) \\
\quad+\int_{0}^{\infty} \varphi\left(m_{k-1}, \chi_{k-1}, r\right)\left(\mathfrak{s}_{r}\left[\varepsilon_{k}\right]-\mathfrak{s}_{r}\left[\varepsilon_{k-1}\right]\right) \mathrm{d} r \mid \\
\leq C\left(\left|m_{k}-m_{k-1}\right|+\left|\chi_{k}-\chi_{k-1}\right|+\int_{0}^{t}\left|\dot{\varepsilon}_{k}(\tau)-\dot{\varepsilon}_{k-1}(\tau)\right| \mathrm{d} \tau\right),
\end{aligned}
$$

where we have by (5.6), Proposition 2.2 (ii), Hypothesis 4.1 (i), (iii), (vi), (2.9), (5.5) and the discrete version of the Hölder inequality that 


$$
\begin{aligned}
\left|m_{k}-m_{k-1}\right| & \leq C \int_{0}^{t}\left(\left|\dot{\chi}_{k}-\dot{\chi}_{k-1}\right|+\frac{1}{n} \sum_{k=1}^{n-1}\left|\lambda_{k-j}-\lambda_{k-j-1}\right| D_{j}(\tau)\right) \mathrm{d} \tau \\
& \leq C \int_{0}^{t}\left(\left|\dot{\chi}_{k}-\dot{\chi}_{k-1}\right|+\frac{1}{n} \sum_{k=1}^{n-1}\left|\dot{\varepsilon}_{j}\right|\left|\lambda_{k-j}-\lambda_{k-j-1}\right|\right) \mathrm{d} \tau \\
& \leq C\left(\int_{0}^{t}\left|\dot{\chi}_{k}-\dot{\chi}_{k-1}\right| \mathrm{d} \tau+\frac{1}{n^{2}} \int_{0}^{t} \sum_{k=1}^{n-1}\left|\dot{\varepsilon}_{j}\right| \mathrm{d} \tau\right)
\end{aligned}
$$

Moreover by (5.5), Proposition 2.2 (ii) and Hypothesis 4.1 (i) we have

$$
\begin{aligned}
& \left|\dot{\chi}_{k}-\dot{\chi}_{k-1}\right|+\frac{\mathrm{d}}{\mathrm{d} t}\left|\chi_{k}-\chi_{k-1}\right| \leq C\left(\left|\theta_{k}-\theta_{k-1}\right|\right. \\
& \left.+\int_{0}^{\infty}\left(\varphi_{\chi}\left(m_{k}, \chi_{k}, r\right) \mathfrak{s}_{r}^{2}\left[\varepsilon_{k}\right](\tau)-\varphi_{\chi}\left(m_{k-1}, \chi_{k-1}, r\right) \mathfrak{s}_{r}^{2}\left[\varepsilon_{k-1}\right](\tau)\right) \mathrm{d} r\right) \\
\leq & C\left[\left|\theta_{k}-\theta_{k-1}\right|+\int_{0}^{\infty}\left(\mid \varphi_{\chi}\left(m_{k}, \chi_{k}, r\right)-\varphi_{\chi}\left(m_{k-1}, \chi_{k}, r\right)+\varphi_{\chi}\left(m_{k-1}, \chi_{k}, r\right)\right.\right. \\
& \left.\left.-\varphi_{\chi}\left(m_{k-1}, \chi_{k-1}, r\right) \mid \mathfrak{s}_{r}^{2}\left[\varepsilon_{k}\right](t)+\varphi_{\chi}\left(m_{k-1}, \chi_{k-1}, r\right)\left(\mathfrak{s}_{r}^{2}\left[\varepsilon_{k}\right]-\mathfrak{s}_{r}^{2}\left[\varepsilon_{k-1}\right]\right)(t)\right) \mathrm{d} r\right] \\
\leq & C\left(\left|\theta_{k}-\theta_{k-1}\right|+\left|m_{k}-m_{k-1}\right|+\left|\chi_{k}-\chi_{k-1}\right|+\int_{0}^{\infty} \varphi_{\chi}\left(m_{k-1}, \chi_{k-1}, r\right)\left(\mathfrak{s}_{r}^{2}\left[\varepsilon_{k}\right]-\mathfrak{s}_{r}^{2}\left[\varepsilon_{k-1}\right]\right)(t) \mathrm{d} r\right) .
\end{aligned}
$$

After integrating, we estimate the second term on the left hand side using Hypothesis 4.1 (i), Proposition 2.2 (iii) and finally Gronwall's lemma as

$$
\left|\chi_{k}-\chi_{k-1}\right| \leq\left|\chi_{k}^{0}-\chi_{k-1}^{0}\right|+C \int_{0}^{t}\left(\left|\theta_{k}-\theta_{k-1}\right|+\left|m_{k}-m_{k-1}\right|+\int_{0}^{\tau}\left|\dot{\varepsilon}_{k}\left(\tau^{\prime}\right)-\dot{\varepsilon}_{k-1}\left(\tau^{\prime}\right)\right| \mathrm{d} \tau^{\prime}\right) \mathrm{d} \tau .
$$

Plugging this back into the right hand side of (6.32) we have

$$
\begin{aligned}
\left|\dot{\chi}_{k}-\dot{\chi}_{k-1}\right|+\frac{\mathrm{d}}{\mathrm{d} t}\left|\chi_{k}-\chi_{k-1}\right| \leq & C\left(\left|\chi_{k}^{0}-\chi_{k-1}^{0}\right|+\left|\theta_{k}-\theta_{k-1}\right|+\left|m_{k}-m_{k-1}\right|\right) \\
& +\int_{0}^{t}\left(\left|\theta_{k}-\theta_{k-1}\right|+\left|m_{k}-m_{k-1}\right|+\left|\dot{\varepsilon}_{k}-\dot{\varepsilon}_{k-1}\right|\right) \mathrm{d} \tau .
\end{aligned}
$$

Integrating (6.34) in time and exploiting (6.15), estimate (6.31) becomes

$$
\begin{aligned}
\left|m_{k}-m_{k-1}\right| & \leq C\left(\int_{0}^{t}\left|\dot{\chi}_{k}-\dot{\chi}_{k-1}\right| \mathrm{d} \tau+\frac{1}{n^{2}} \int_{0}^{t} \sum_{k=1}^{n-1}\left|\dot{\varepsilon}_{j}\right| \mathrm{d} \tau\right) \\
& \leq C\left(\left|\chi_{k}^{0}-\chi_{k-1}^{0}\right|+\int_{0}^{t}\left(\left|\theta_{k}-\theta_{k-1}\right|+\left|m_{k}-m_{k-1}\right|+\left|\dot{\varepsilon}_{k}-\dot{\varepsilon}_{k-1}\right|\right) \mathrm{d} \tau+\frac{1}{n}\right),
\end{aligned}
$$

We now integrate (6.21) in time. The bound for the initial condition

$$
\frac{1}{2 n} \sum_{k=1}^{n-1} \dot{\varepsilon}_{k}^{2}(0)+n \sum_{k=1}^{n}\left(\varepsilon_{k}-\varepsilon_{k-1}\right)^{2}(0)+n \sum_{k=1}^{n}\left(\dot{w}_{k}-\dot{w}_{k-1}\right)^{2}(0) \leq C,
$$

can be derived as follows: for the last term, we use (6.11); for the second term we use the initial condition $w(x, 0)=0$ and (5.3); finally for the first one, we consider equation (5.2) for $t=0$ (together with the initial 
conditions (5.7)) and test it by $\dot{\varepsilon}_{k}(0)$, then we use once more (6.11). At this point, we conclude from the above considerations that

$$
\begin{aligned}
& \frac{1}{2 n} \sum_{k=1}^{n-1} \dot{\varepsilon}_{k}^{2}(t)+n \sum_{k=1}^{n}\left(\varepsilon_{k}-\varepsilon_{k-1}\right)^{2}(t)+\int_{0}^{t} n \sum_{k=1}^{n}\left(\dot{\varepsilon}_{k}-\dot{\varepsilon}_{k-1}\right)^{2}(\tau) \mathrm{d} \tau \\
& \leq C\left(1+\frac{1}{n} \sum_{k=1}^{n-1} \theta_{k}^{2}(t)+\left(\int_{0}^{t} \frac{1}{n} \sum_{k=1}^{n-1} \dot{\theta}_{k}^{2}(\tau) \mathrm{d} \tau\right)^{1 / 2}+\int_{0}^{t} n \sum_{k=1}^{n}\left(\theta_{k}-\theta_{k-1}\right)^{2}(\tau) \mathrm{d} \tau\right. \\
& \left.+\int_{0}^{t} \int_{0}^{\tau} n \sum_{k=1}^{n}\left(\dot{\varepsilon}_{k}-\dot{\varepsilon}_{k-1}\right)^{2}\left(\tau^{\prime}\right) \mathrm{d} \tau^{\prime} \mathrm{d} \tau\right)
\end{aligned}
$$

Gronwall's argument and (6.14) then yields

$$
\begin{aligned}
& \frac{1}{2 n} \sum_{k=1}^{n-1} \dot{\varepsilon}_{k}^{2}(t)+n \sum_{k=1}^{n}\left(\varepsilon_{k}-\varepsilon_{k-1}\right)^{2}(t)+\int_{0}^{t} n \sum_{k=1}^{n}\left(\dot{\varepsilon}_{k}-\dot{\varepsilon}_{k-1}\right)^{2}(\tau) \mathrm{d} \tau \\
& \leq C\left(1+\left(\int_{0}^{t} \frac{1}{n} \sum_{k=1}^{n-1} \dot{\theta}_{k}^{2}(\tau) \mathrm{d} \tau\right)^{1 / 2}+\int_{0}^{t} n \sum_{k=1}^{n}\left(\theta_{k}-\theta_{k-1}\right)^{2}(\tau) \mathrm{d} \tau\right) .
\end{aligned}
$$

We need now more regularity for the temperature. To this aim we now test (5.4) by $\theta_{k}$. The presence of the additional term in (5.4) this time provides no further difficulties, due to the fact that the term $\left(\mathcal{M}_{k}-L\right)$ is bounded (in absolute value), therefore we can use Hypothesis 2.1 (i) and (vii) and (6.22)-(6.24) together with (2.9) to obtain

$$
\frac{\mathrm{d}}{\mathrm{d} t}\left(\frac{1}{n} \sum_{k=1}^{n-1} \theta_{k}^{2}\right)+n \sum_{k=1}^{n}\left(\theta_{k}-\theta_{k-1}\right)^{2} \leq C\left(1+\frac{1}{n} \sum_{k=1}^{n-1}\left(\dot{\varepsilon}_{k}^{2}+\theta_{k}+\left|\dot{\varepsilon}_{k}\right|\left(1+\theta_{k}\right)\right) \theta_{k}\right) .
$$

The estimate of the right hand side of the previous inequality follows exactly as in [13], getting at the end

$$
\frac{1}{n} \sum_{k=1}^{n-1} \theta_{k}^{2}(t)+\int_{0}^{t} n \sum_{k=1}^{n}\left(\theta_{k}-\theta_{k-1}\right)^{2}(\tau) \mathrm{d} \tau \leq C\left(1+\left(\int_{0}^{t} \frac{1}{n} \sum_{k=1}^{n-1} \dot{\varepsilon}_{k}^{4} \mathrm{~d} \tau\right)^{1 / 2}\right) .
$$

Finally, we test (5.4) by $\dot{\theta}_{k}$ and obtain from Hölder's inequality that (also in this case the additional term brings no major difficulties)

$$
\int_{0}^{\tau} \frac{1}{n} \sum_{k=1}^{n-1} \dot{\theta}_{k}^{2}\left(\tau^{\prime}\right) \mathrm{d} \tau^{\prime}+n \sum_{k=1}^{n}\left(\theta_{k}-\theta_{k-1}\right)^{2}(\tau) \leq C_{1}\left(1+\int_{0}^{t} \frac{1}{n} \sum_{k=1}^{n-1} \dot{\varepsilon}_{k}^{4} \mathrm{~d} \tau+\int_{0}^{t} \frac{1}{n} \sum_{k=1}^{n-1} \theta_{k}^{4} \mathrm{~d} \tau\right)
$$

for any $\tau \in[0, t]$ and for some $C_{1}>0$ that will be needed in a moment. The last term in the right hand side can be estimated using the discrete Gagliardo-Nirenberg inequality

$$
|\mathbf{v}|_{q} \leq C\left(|\mathbf{v}|_{s}+|\mathbf{v}|_{s}^{1-\varrho}|\mathbf{D v}|_{p}^{\varrho}\right), \quad \varrho=\frac{\frac{1}{s}-\frac{1}{q}}{1+\frac{1}{s}-\frac{1}{p}}, \quad \mathbf{v}=\left(v_{0}, v_{1}, \ldots, v_{n}\right),
$$

where we set

$$
|\mathbf{v}|_{p}:=\left(\frac{1}{n} \sum_{k=0}^{n}\left|v_{k}\right|^{p}\right)^{1 / p}, \quad|\mathbf{D v}|_{p}:=\left(n^{p-1} \sum_{k=1}^{n}\left|v_{k}-v_{k-1}\right|^{p}\right)^{1 / p} \quad \text { if } 1 \leq p<\infty
$$


and

$$
|\mathbf{v}|_{\infty}:=\max _{k=1, \ldots, n}\left|v_{k}\right| \quad \text { if } p=\infty
$$

Applying (6.39) with the choices $q=\infty, s=1, p=2, \varrho=2 / 3$, we get

$$
\begin{aligned}
\int_{0}^{t} \frac{1}{n} \sum_{k=1}^{n-1} \theta_{k}^{4} \mathrm{~d} \tau & \leq C \max _{\tau \in[0, t]} \max _{i=1, \ldots, n} \theta_{i}^{4 / 3}(\tau) \leq C \max _{\tau \in[0, t]}\left(1+\left(n \sum_{k=1}^{n}\left(\theta_{k}-\theta_{k-1}\right)^{2}(\tau)\right)^{4 / 9}\right) \\
& \leq C+\frac{4}{9 C_{1}}\left(\max _{\tau \in[0, t]} n \sum_{k=1}^{n}\left(\theta_{k}-\theta_{k-1}\right)^{2}(\tau)\right)
\end{aligned}
$$

with $C_{1}$ as in (6.38), and where we employed also (6.11), (6.14) and Young's inequality. Passing to the $\max _{\tau \in[0, t]}$ in (6.38) and absorbing the last term of the previous inequality in the left hand side, we thus obtain

$$
\int_{0}^{t} \frac{1}{n} \sum_{k=1}^{n-1} \dot{\theta}_{k}^{2}(\tau) \mathrm{d} \tau+n \sum_{k=1}^{n}\left(\theta_{k}-\theta_{k-1}\right)^{2}(t) \leq C\left(1+\int_{0}^{t} \frac{1}{n} \sum_{k=1}^{n-1} \dot{\varepsilon}_{k}^{4} \mathrm{~d} \tau\right)
$$

which in turn gives, by (6.36),

$$
\frac{1}{n} \sum_{k=1}^{n-1} \dot{\varepsilon}_{k}^{2}(t)+n \int_{0}^{t} \sum_{k=1}^{n}\left(\dot{\varepsilon}_{k}-\dot{\varepsilon}_{k-1}\right)^{2}(\tau) \mathrm{d} \tau \leq C\left(1+\left(\int_{0}^{t} \frac{1}{n} \sum_{k=1}^{n-1} \dot{\varepsilon}_{k}^{4} \mathrm{~d} \tau\right)^{1 / 2}\right) .
$$

The last term in the right hand side can be estimated using once more the Gagliardo Nirenberg inequality (6.39) with the choices $q=4, p=s=2, \varrho=1 / 4$. Absorbing the resulting terms in the left hand side, we finally get that there exists a constant $C>0$ such that

$$
\begin{array}{r}
\frac{1}{n} \sum_{k=1}^{n-1} \dot{\varepsilon}_{k}^{2}(t)+\int_{0}^{t} n \sum_{k=1}^{n}\left(\dot{\varepsilon}_{k}-\dot{\varepsilon}_{k-1}\right)^{2}(\tau) \mathrm{d} \tau+\int_{0}^{t} \frac{1}{n} \sum_{k=1}^{n-1}\left(\dot{\varepsilon}_{k}^{4}+\varepsilon_{k}^{4}\right)(\tau) \mathrm{d} \tau \leq C, \\
\int_{0}^{t} \frac{1}{n} \sum_{k=1}^{n-1}\left(\theta_{k}^{4}+\dot{\theta}_{k}^{2}\right)(\tau) \mathrm{d} \tau+n \sum_{k=1}^{n}\left(\theta_{k}-\theta_{k-1}\right)^{2}(t) \leq C,
\end{array}
$$

for $t \in[0, T]$. From (6.41) it follows that

$$
\max _{t \in[0, T]} \max _{i=1, \ldots, n} \theta_{i}(t) \leq C,
$$

hence

$$
\max _{t \in[0, T]} \max _{i=1, \ldots, n}\left|\dot{\chi}_{i}(t)\right| \leq C
$$

and so finally

$$
\max _{t \in[0, T]} \max _{i=1, \ldots, n}\left(\theta_{i}(t)+\left|\dot{\chi}_{i}(t)\right|+\left|\dot{m}_{i}(t)\right|\right) \leq C .
$$

By comparison of the terms in (5.4), we also have

$$
\int_{0}^{t} n^{3} \sum_{k=1}^{n-1}\left(\theta_{k+1}-2 \theta_{k}+\theta_{k-1}\right)^{2}(\tau) \mathrm{d} \tau \leq C,
$$


and similarly for $u_{k}$ by (5.2). Finally, we differentiate (5.1) once in $t$ and test by $\ddot{\varepsilon}_{k},(5.2)$ twice in $t$ and test by $\ddot{w}_{k}$, and sum the two equations up. The dependence of $P$ upon $\chi_{k}$ this time brings no additional problems due to the fact that we already estimated this term in (6.25). Therefore using (6.40)-(6.41) and treating the initial conditions as in [13], we get the final estimate

$$
\frac{1}{n} \sum_{k=1}^{n-1} \ddot{w}_{k}^{2}(t)+n \sum_{k=1}^{n}\left(\ddot{w}_{k}-\ddot{w}_{k-1}\right)^{2}(t)+\int_{0}^{t} \frac{1}{n} \sum_{k=1}^{n-1} \ddot{\varepsilon}_{k}^{2}(\tau) \mathrm{d} \tau \leq C .
$$

\section{Passage to the Limit}

Let us set $\Delta_{k} \varphi=n\left(\varphi_{k}-\varphi_{k-1}\right)$ and $\Delta_{k}^{2} \varphi=n^{2}\left(\varphi_{k+1}-2 \varphi_{k}+\varphi_{k-1}\right)$, for a given sequence $\left\{\varphi_{k}: k=\right.$ $0,1, \ldots, n\}$ with the aim to define correspondingly piecewise constant, piecewise linear and piecewise quadratic interpolations

$$
\begin{aligned}
& \bar{\varphi}^{(n)}(x)= \begin{cases}\varphi_{k} & \text { for } x \in\left[\frac{k-1}{n}, \frac{k}{n}\right), k=1, \ldots, n-1, \\
\varphi_{n-1} & \text { for } x \in\left[\frac{n-1}{n}, 1\right]\end{cases} \\
& \hat{\varphi}^{(n)}(x)=\varphi_{k-1}+\left(x-\frac{k-1}{n}\right) \Delta_{k} \varphi \quad \text { for } \quad x \in\left[\frac{k-1}{n}, \frac{k}{n}\right), \quad k=1, \ldots, n, \\
& \tilde{\varphi}^{(n)}(x)=\left\{\begin{array}{l}
\frac{1}{2}\left(\varphi_{k-1}+\varphi_{k}\right)+\left(x-\frac{k-1}{n}\right) \Delta_{k} \varphi+\frac{1}{2}\left(x-\frac{k-1}{n}\right)^{2} \Delta_{k}^{2} \varphi \\
\text { for } x \in\left[\frac{k-1}{n}, \frac{k}{n}\right), k=1, \ldots, n-1, \\
\frac{1}{2}\left(\varphi_{n-1}+\varphi_{n}\right)+\left(x-\frac{n-1}{n}\right) \Delta_{n} \varphi+\frac{1}{2}\left(x-\frac{n-1}{n}\right)^{2} \Delta_{n-1}^{2} \varphi \\
\text { for } x \in\left[\frac{n-1}{n}, 1\right] .
\end{array}\right.
\end{aligned}
$$

We also define

$$
\lambda^{(n)}(x, y)=\lambda_{k-j} \quad \text { for }(x, y) \in\left[\frac{k-1}{n}, \frac{k}{n}\right) \times\left[\frac{j-1}{n}, \frac{j}{n}\right) .
$$

In the previous section we derived a priori estimates for the functions $\bar{\varepsilon}^{(n)}, \bar{\theta}^{(n)}, \bar{u}^{(n)}, \bar{w}^{(n)}, \bar{\chi}^{(n)}, \bar{m}^{(n)}, \hat{\varepsilon}^{(n)}$, $\tilde{\theta}^{(n)}, \tilde{u}^{(n)}, \tilde{w}^{(n)},(6.40)-(6.44)$ that we rewrite in the form

$$
\begin{array}{r}
\left|\bar{\varepsilon}_{t}^{(n)}(t)\right|_{2}^{2}+\int_{0}^{t}\left|\hat{\varepsilon}_{x t}^{(n)}(\tau)\right|_{2}^{2} \mathrm{~d} \tau+\int_{0}^{t}\left(\left|\bar{\varepsilon}_{t}^{(n)}(\tau)\right|_{4}^{4}+\left|\bar{\varepsilon}^{(n)}(\tau)\right|_{4}^{4}\right) \mathrm{d} \tau \leq C, \\
\int_{0}^{t}\left(\left|\bar{\theta}_{t}^{(n)}(\tau)\right|_{2}^{2}+\left|\bar{\theta}^{(n)}(\tau)\right|_{4}^{4}\right) \mathrm{d} \tau+\left|\hat{\theta}_{x}^{(n)}(t)\right|_{2}^{2} \leq C, \\
\int_{0}^{t}\left|\tilde{\theta}_{x x}^{(n)}(\tau)\right|_{2}^{2} \mathrm{~d} \tau \leq C, \\
\left|\bar{w}_{t t}^{(n)}(t)\right|_{2}^{2}+\left|\hat{w}_{x t t}^{(n)}(t)\right|_{2}^{2}+\int_{0}^{t}\left|\bar{\varepsilon}_{t t}^{(n)}(\tau)\right|_{2}^{2} \mathrm{~d} \tau \leq C, \\
\left|\bar{\theta}(t)^{(n)}\right|_{\infty}+\left|\bar{\chi}_{t}(t)^{(n)}\right|_{\infty}+\left|\bar{m}_{t}(t)^{(n)}\right|_{\infty} \leq C ;
\end{array}
$$

moreover by (5.2)-(5.3),

$$
\int_{0}^{t}\left(\left|\tilde{w}_{x x t}^{(n)}(\tau)\right|_{2}^{2}+\left|\tilde{u}_{x x t}^{(n)}(\tau)\right|_{2}^{2}\right) \mathrm{d} \tau \leq C
$$


and by (5.8), (6.42)

$$
\int_{0}^{t}\left|\hat{\varepsilon}_{t t}(\tau)\right|_{2}^{2} \mathrm{~d} \tau \leq C\left(1+\int_{0}^{t} \frac{2}{n} \sum_{k=1}^{n-1}\left(\ddot{\varepsilon}_{k}^{2}+\dot{\theta}_{k}^{2}\right)(\tau) \mathrm{d} \tau\right) \leq C .
$$

On the other hand, the system (5.1)-(5.5) can be expressed in the form

$$
\begin{aligned}
\bar{u}_{t}^{(n)} & =\bar{\varepsilon}^{(n)}+P\left[\bar{m}^{(n)}, \bar{\chi}^{(n)}, \bar{\varepsilon}^{(n)}\right]+\bar{\varepsilon}_{t}^{(n)}-\left(\bar{\theta}^{(n)}-\theta_{\mathrm{ref}}\right), \\
\bar{w}_{t}^{(n)}-\bar{\varepsilon}_{t}^{(n)} & =-\tilde{u}_{x x}^{(n)}+\bar{f}^{(n)} \\
\bar{\varepsilon}^{(n)} & =\tilde{w}_{x x}^{(n)} \\
\bar{\theta}_{t}^{(n)} & =\tilde{\theta}_{x x}^{(n)}+\bar{m}_{t}^{(n)} \bar{K}^{(n)}+\bar{\chi}_{t}^{(n)} \overline{\mathcal{M}}^{(n)}+\bar{D}^{(n)}+\left(\bar{\varepsilon}_{t}^{(n)}\right)^{2}-\bar{\theta}^{(n)} \bar{\varepsilon}_{t}^{(n)} \\
& +\bar{g}^{(n)}\left(\bar{\theta}^{(n)}\right)-L \bar{\chi}_{t}^{(n)} \\
\bar{\chi}^{(n)}(x, t) & =\mathfrak{s}_{[0,1]}\left[\bar{\chi}^{(n)}(0), \bar{A}^{(n)}(x, \cdot)\right](t), \\
\bar{m}^{(n)}(x, t) & =\mathfrak{s}_{[0, \infty)}\left[0, \bar{S}^{(n)}(x, \cdot)\right](t), \\
\bar{A}^{(n)}(x, t) & =\int_{0}^{t} \frac{1}{\gamma}\left(\frac{L}{\theta_{\text {ref }}}\left(\bar{\theta}^{(n)}-\theta_{\mathrm{ref}}\right)+\overline{\mathcal{M}}^{(n)}\right)(x, \tau) \mathrm{d} \tau, \\
\overline{\mathcal{M}}^{(n)}(x, t) & =-\frac{1}{2} \int_{0}^{\infty} \varphi_{\chi}\left(\bar{m}^{(n)}, \bar{\chi}^{(n)}, r\right) \mathfrak{s}_{r}^{2}\left[\bar{\varepsilon}^{(n)}\right](x, t) \mathrm{d} r, \\
\bar{S}^{(n)}(x, t) & =\int_{0}^{t}\left(-h\left(\bar{\chi}_{t}^{(n)}(x, \tau)\right)+\int_{0}^{1} \lambda^{(n)}(x, y) \bar{D}^{(n)}(y, \tau) \mathrm{d} y\right)(x, \tau) \mathrm{d} \tau, \\
\bar{D}^{(n)}(x, t) & =\int_{0}^{\infty} \varphi\left(\bar{m}^{(n)}, \bar{\chi}^{(n)}, r\right) \mathfrak{s}_{r}\left[\bar{\varepsilon}^{(n)}\right]\left(\bar{\varepsilon}^{(n)}-\mathfrak{s}_{r}\left[\bar{\varepsilon}^{(n)}\right]\right)_{t}(x, t) \mathrm{d} r, \\
\bar{K}^{(n)}(x, t) & =-\frac{1}{2} \int_{0}^{\infty} \varphi_{m}\left(\bar{m}^{(n)}, \bar{\chi}^{(n)}, r\right) \mathfrak{s}_{r}^{2}\left[\bar{\varepsilon}^{(n)}\right] \mathrm{d} r,
\end{aligned}
$$

with $\bar{\chi}^{(n)}(0)$ chosen according to (5.7). Sobolev embedding theorems together with (7.5), (7.11) allow us to infer the existence of a function $\varepsilon \in W^{1,2}\left(\Omega_{T}\right)$ such that $\varepsilon_{x t}, \varepsilon_{t t} \in L^{2}\left(\Omega_{T}\right)$, and a subsequence of $\left\{\hat{\varepsilon}^{(n)}\right\}$, still indexed by $n$, such that

$$
\hat{\varepsilon}^{(n)} \rightarrow \varepsilon \text { strongly in } C\left(\bar{\Omega}_{T}\right), \quad \hat{\varepsilon}_{t}^{(n)} \rightarrow \varepsilon_{t} \text { strongly in } L^{p}\left(\Omega_{T}\right)
$$

for all $p>1$. Moreover

$$
\left|\bar{\varepsilon}_{t}^{(n)}-\hat{\varepsilon}_{t}^{(n)}\right|^{2}(x, t) \leq\left|\dot{\varepsilon}_{k}-\dot{\varepsilon}_{k-1}\right|^{2}(t)
$$

for $x \in[(k-1) / n, k / n]$, so that

$$
\int_{0}^{t} \int_{0}^{1}\left|\bar{\varepsilon}_{t}^{(n)}-\hat{\varepsilon}_{t}^{(n)}\right|^{2}(x, \tau) \mathrm{d} x \mathrm{~d} \tau \leq \int_{0}^{t} \frac{1}{n} \sum_{k=1}^{n}\left(\dot{\varepsilon}_{k}-\dot{\varepsilon}_{k-1}\right)^{2}(\tau) \mathrm{d} \tau \leq \frac{C}{n^{2}}
$$

and

$$
\bar{\varepsilon}_{t}^{(n)} \rightarrow \varepsilon_{t} \text { strongly in } L^{2}\left(\Omega_{T}\right) .
$$

In an analogous way we can deduce that

$$
\bar{\varepsilon}^{(n)} \rightarrow \varepsilon \text { strongly in } L^{\infty}\left(\Omega_{T}\right)
$$


and we can also infer the existence of functions $u, w, \theta \in C\left(\bar{\Omega}_{T}\right)$ such that, up to subsequences when necessary,

$$
\left.\begin{array}{l}
\tilde{w}_{x x t}^{(n)} \rightarrow \varepsilon_{t}=w_{x x t}, \tilde{u}_{x x}^{(n)} \rightarrow u_{x x} \text { strongly in } L^{2}\left(\Omega_{T}\right), \\
\tilde{\theta}_{x x}^{(n)} \rightarrow \theta_{x x} \text { weakly in } L^{2}\left(\Omega_{T}\right), \quad \bar{\theta}^{(n)} \rightarrow \theta \text { strongly in } L^{\infty}\left(\Omega_{T}\right) .
\end{array}\right\}
$$

The main complications come when dealing with the phase terms. This time indeed we have by Proposition 2.2 (iii), for all $n, l \in \mathbb{N}$

$$
\begin{aligned}
& \left|\bar{\chi}^{(n)}-\bar{\chi}^{(l)}\right|(x, t) \leq \int_{0}^{t}\left|\bar{\chi}_{t}^{(n)}-\bar{\chi}_{t}^{(l)}\right|(x, \tau) \mathrm{d} \tau \\
\leq & C\left[\int_{0}^{t}\left(\left|\bar{\theta}^{(n)}-\bar{\theta}^{(l)}\right|+\left|\bar{m}^{(n)}-\bar{m}^{(l)}\right|\right)(x, \tau) \mathrm{d} \tau+\max _{\tau \in[0, t]}\left|\bar{\varepsilon}^{(n)}-\bar{\varepsilon}^{(l)}\right|(x, \tau)\right]+\left|\bar{\chi}^{(n)}-\bar{\chi}^{(l)}\right|(x, 0) .
\end{aligned}
$$

On the other hand, by Proposition 2.2 (ii) and by (7.17)-(7.21) we have, for all $x \in(0,1)$ that

$$
\begin{aligned}
\left|\bar{m}^{(n)}-\bar{m}^{(l)}\right|(x, t) \leq & \int_{0}^{t}\left|\bar{m}_{t}^{(n)}-\bar{m}_{t}^{(l)}\right|(x, \tau) \mathrm{d} \tau \\
\leq & C \int_{0}^{t}\left|\bar{\chi}_{t}^{(n)}-\bar{\chi}_{t}^{(l)}\right|(x, \tau) \mathrm{d} \tau \\
& +\int_{0}^{t} \int_{0}^{1} \int_{0}^{\infty} \mid \lambda^{(n)}(x, y) \varphi\left(\bar{m}^{(n)}, \bar{\chi}^{(n)}, r\right) \delta^{(n)}(y, t, r) \\
& -\lambda^{(l)}(x, y) \varphi\left(\bar{m}^{(l)}, \bar{\chi}^{(l)}, r\right) \delta^{(l)}(y, t, r) \mid \mathrm{d} r \mathrm{~d} y \mathrm{~d} \tau
\end{aligned}
$$

where we denote

$$
\delta^{(n)}=\delta^{(n)}(y, t, r)=\mathfrak{s}_{r}\left[\bar{\varepsilon}^{(n)}\right]\left(\bar{\varepsilon}^{(n)}-\mathfrak{s}_{r}\left[\bar{\varepsilon}^{(n)}\right]\right)_{t}(y, t)=r\left|\mathfrak{p}_{r}\left[\bar{\varepsilon}^{(n)}\right]_{t}(y, t)\right|
$$

Now (7.27) implies

$$
\begin{aligned}
& \left|\bar{m}^{(n)}-\bar{m}^{(l)}\right|(x, t) \leq C \int_{0}^{t}\left|\bar{m}_{t}^{(n)}-\bar{m}_{t}^{(l)}\right|(x, \tau) \mathrm{d} \tau \\
\leq & C\left[\int_{0}^{t}\left(\left|\bar{\theta}^{(n)}-\bar{\theta}^{(l)}\right|+\left|\bar{m}^{(n)}-\bar{m}^{(l)}\right|\right)(x, \tau) \mathrm{d} \tau+\max _{\tau \in[0, t]}\left|\bar{\varepsilon}^{(n)}-\bar{\varepsilon}^{(l)}\right|(x, \tau)\right]+\left|\bar{\chi}^{(n)}-\bar{\chi}^{(l)}\right|(x, 0) \\
& +\int_{0}^{t} \int_{0}^{1} \int_{0}^{\infty} \lambda^{(n)}(x, y) \varphi\left(\bar{m}^{(n)}, \bar{\chi}^{(n)}, r\right)\left|\delta^{(n)}-\delta^{(l)}\right|(y, t, r) \mathrm{d} r \mathrm{~d} y \mathrm{~d} \tau \\
& +\int_{0}^{t} \int_{0}^{1} \int_{0}^{\infty} \lambda^{(n)}(x, y)\left|\varphi\left(\bar{m}^{(n)}, \bar{\chi}^{(n)}, r\right)-\varphi\left(\bar{m}^{(l)}, \bar{\chi}^{(l)}, r\right)\right| \delta^{(l)}(y, t, r) \mathrm{d} r \mathrm{~d} y \mathrm{~d} \tau \\
& +\int_{0}^{t} \int_{0}^{1} \int_{0}^{\infty}\left|\lambda^{(n)}(x, y)-\lambda^{(l)}(x, y)\right| \varphi\left(\bar{m}^{(l)}, \bar{\chi}^{(l)}, r\right) \mid \delta^{(l)}(y, t, r) \mathrm{d} r \mathrm{~d} y \mathrm{~d} \tau .
\end{aligned}
$$

Proposition 2.2 (ii) allows us to conclude that

$$
\int_{0}^{t}\left|\delta^{(n)}-\delta^{(l)}\right|(y, \tau) \mathrm{d} \tau \leq r \int_{0}^{t}\left|\bar{\varepsilon}_{t}^{(n)}-\bar{\varepsilon}_{t}^{(l)}\right|(y, \tau) \mathrm{d} \tau
$$


hence

$$
\int_{0}^{t} \int_{0}^{1} \int_{0}^{\infty} \lambda^{(n)}(x, y) \varphi\left(\bar{m}^{(n)}, \bar{\chi}^{(n)}, r\right)\left|\delta^{(n)}-\delta^{(l)}\right| \mathrm{d} r \mathrm{~d} y \mathrm{~d} \tau \leq C \int_{0}^{t} \int_{0}^{1}\left|\bar{\varepsilon}_{t}^{(n)}-\bar{\varepsilon}_{t}^{(l)}\right|(y, \tau) \mathrm{d} y \mathrm{~d} \tau
$$

by Hypothesis 4.1 (i), (iii). In a similar way, exploiting again Hypothesis 4.1 (i), we have

$$
\begin{aligned}
& \int_{0}^{t} \int_{0}^{1} \int_{0}^{\infty} \delta^{(l)} \lambda^{(n)}(x, y)\left|\varphi\left(\bar{m}^{(n)}, \bar{\chi}^{(n)}, r\right)-\varphi\left(\bar{m}^{(l)}, \bar{\chi}^{(l)}, r\right)\right| \mathrm{d} r \mathrm{~d} y \mathrm{~d} \tau \\
\leq & C \int_{0}^{t}\left(\int_{0}^{1}\left|\bar{\varepsilon}_{t}^{(l)}(y, \tau)\right| \mathrm{d} y\right) \max _{x \in(0,1)}\left(\left|m^{(n)}(x, \tau)-m^{(l)}(x, \tau)\right|+\left|\chi^{(n)}(x, \tau)-\chi^{(l)}(x, \tau)\right|\right) \mathrm{d} \tau .
\end{aligned}
$$

Combining (7.26)-(7.31) and the pointwise bound

$$
\left|\lambda^{(n)}(x, y)-\lambda^{(l)}(x, y)\right| \leq \frac{4 \Lambda}{\min \{n, l\}}
$$

we obtain the following inequality:

$$
\begin{aligned}
& \max _{x \in(0,1)}\left(\left|\bar{m}^{(n)}-\bar{m}^{(l)}\right|(x, t)+\left|\bar{\chi}^{(n)}-\bar{\chi}^{(l)}\right|(x, t)\right) \\
\leq & \max _{x \in(0,1)} \int_{0}^{t}\left(\left|\bar{m}_{t}^{(n)}-\bar{m}_{t}^{(l)}\right|(x, \tau)+\left|\bar{\chi}_{t}^{(n)}-\bar{\chi}_{t}^{(l)}\right|(x, \tau)\right) \mathrm{d} \tau \\
\leq & q_{n l}+C \int_{0}^{t}\left(\int_{0}^{1}\left|\bar{\varepsilon}_{t}^{(l)}(y, \tau)\right| \mathrm{d} y\right) \max _{x \in(0,1)}\left(\left|\bar{m}^{(n)}-\bar{m}^{(l)}\right|(x, \tau)+\left|\bar{\chi}^{(n)}-\bar{\chi}^{(l)}\right|(x, \tau)\right) \mathrm{d} \tau,
\end{aligned}
$$

with

$$
q_{n l}=C\left(\frac{1}{\min \{n, l\}}+\left|\bar{\chi}^{(n)}(\cdot, 0)-\bar{\chi}^{(l)}(\cdot, 0)\right|_{\infty}+\left\|\bar{\theta}^{(n)}-\bar{\theta}^{(l)}\right\|_{\infty}+\left\|\bar{\varepsilon}_{t}^{(n)}-\bar{\varepsilon}_{t}^{(l)}\right\|_{1}+\left\|\bar{\varepsilon}^{(n)}-\bar{\varepsilon}^{(l)}\right\|_{\infty}\right) .
$$

Arguing as in [13], it is possible to prove that $q_{n l}$ is small if $n, l$ are large, hence, $\bar{m}^{(n)}$ and $\bar{\chi}^{(n)}$ are Cauchy sequences, and this gives

$$
\begin{aligned}
& \bar{m}^{(n)} \rightarrow m \text { strongly in } L^{\infty}\left(\Omega_{T}\right), \\
& \bar{\chi}^{(n)} \rightarrow \chi \text { strongly in } L^{\infty}\left(\Omega_{T}\right),
\end{aligned}
$$

and, by (7.32),

$$
\begin{aligned}
& \bar{m}_{t}^{(n)} \rightarrow m_{t} \text { strongly in } L^{\infty}\left(0,1 ; L^{1}(0, T)\right) \\
& \bar{\chi}_{t}^{(n)} \rightarrow \chi_{t} \text { strongly in } L^{\infty}\left(0,1 ; L^{1}(0, T)\right) .
\end{aligned}
$$

By virtue of $(6.42), \bar{m}_{t}^{(n)}$ and $\bar{\chi}_{t}^{(n)}$ are uniformly bounded in $L^{\infty}\left(\Omega_{T}\right)$, hence

$$
\bar{m}_{t}^{(n)} \rightarrow m_{t}, \bar{\chi}_{t}^{(n)} \rightarrow \chi_{t} \text { in } L^{\infty}\left(\Omega_{T}\right) \text { weakly star. }
$$

Also in this case when the Prandtl-Ishlinskii density function depends on $\chi$, using the convergences (7.23), (7.24), and Proposition 2.2, we are able to conclude that $\bar{D}^{(n)}(x, \cdot), \bar{K}^{(n)}(x, \cdot)$ converge for all $x \in[0,1]$ to $D[m, \chi, \varepsilon](x, \cdot), \mathcal{K}[m, \chi, \varepsilon](x, \cdot)$, respectively, strongly in $L^{2}(0, T)$. By the Lebesgue Dominated Convergence Theorem, passing in $L^{2}\left(\Omega_{T}\right)$ to the limit in (7.12)-(7.22), we conclude that $(u, w, \theta, m, \chi)$ is a strong solution to 
(4.1)-(4.4) with the regularity indicated in Theorem 4.2 and satisfying the initial conditions (4.6). The boundary conditions (4.7) hold by arguing exactly as in [13].

\section{Proof of the UNIQUeness}

Let $(u, w, \theta, \chi, m),(\tilde{u}, \tilde{w}, \tilde{\theta}, \tilde{\chi}, \tilde{m})$ be two solutions to (4.1)-(4.7), with the regularity as in Theorem 4.2, and with the same initial conditions and the same right hand sides.

We take the difference of (4.3) for $\theta$ and $\tilde{\theta}$ obtaining

$$
\begin{aligned}
& c\left(\theta_{t}-\tilde{\theta}_{t}\right)-\kappa\left(\theta_{x x}-\tilde{\theta}_{x x}\right)=\left(\mathcal{D}\left[m, \chi, w_{x x}\right]-\mathcal{D}\left[\tilde{m}, \tilde{\chi}, \tilde{w}_{x x}\right]\right) \\
& +\nu\left(w_{x x t}^{2}-\tilde{w}_{x x t}^{2}\right)-\beta\left(\theta w_{x x t}-\tilde{\theta} \tilde{w}_{x x t}\right) \\
& -\left(\frac{1}{2} m_{t} \int_{0}^{\infty} \varphi_{m}(m, \chi, r) \mathfrak{s}_{r}^{2}\left[w_{x x}\right] \mathrm{d} r-\frac{1}{2} \tilde{m}_{t} \int_{0}^{\infty} \varphi_{m}(\tilde{m}, \tilde{\chi}, r) \mathfrak{s}_{r}^{2}\left[\tilde{w}_{x x}\right] \mathrm{d} r\right) \\
& -\left(\frac{1}{2} \chi_{t} \int_{0}^{\infty} \varphi_{\chi}(m, \chi, r) \mathfrak{s}_{r}^{2}\left[w_{x x}\right] \mathrm{d} r-\frac{1}{2} \tilde{\chi}_{t} \int_{0}^{\infty} \varphi_{m}(\tilde{m}, \tilde{\chi}, r) \mathfrak{s}_{r}^{2}\left[\tilde{w}_{x x}\right] \mathrm{d} r\right) \\
& -L\left(\chi_{t}-\tilde{\chi}_{t}\right)+g(\theta, x, t)-g(\tilde{\theta}, x, t) .
\end{aligned}
$$

We integrate the previous inequality in time and estimate the terms in the right hand side. First of all we have

$$
\begin{aligned}
& \int_{0}^{t}\left|\mathcal{D}\left[m, \chi, w_{x x}\right]-\mathcal{D}\left[\tilde{m}, \tilde{\chi}, \tilde{w}_{x x}\right]\right|(x, \tau) \mathrm{d} \tau \\
= & \int_{0}^{t}\left|\int_{0}^{\infty} r \varphi(m, \chi, r)\right| \mathfrak{p}_{r}\left[w_{x x}\right]_{t}\left|\mathrm{~d} r-\int_{0}^{\infty} r \varphi(\tilde{m}, \tilde{\chi}, r)\right| \mathfrak{p}_{r}\left[\tilde{w}_{x x}\right]_{t}|\mathrm{~d} r| \mathrm{d} \tau \\
= & \int_{0}^{t}\left|\int_{0}^{\infty} r(\varphi(m, \chi, r)-\varphi(\tilde{m}, \chi, r))\right| \mathfrak{p}_{r}\left[w_{x x}\right]_{t} \mid \mathrm{d} r \\
& +\int_{0}^{\infty} r(\varphi(\tilde{m}, \chi, r)-\varphi(\tilde{m}, \tilde{\chi}, r))\left|\mathfrak{p}_{r}\left[w_{x x}\right]_{t}\right| \mathrm{d} r+\int_{0}^{\infty} r \varphi(\tilde{m}, \tilde{\chi}, r)\left|\mathfrak{p}_{r}\left[w_{x x}\right]_{t}-\mathfrak{p}_{r}\left[\tilde{w}_{x x}\right]_{t}\right| \mathrm{d} r \mid \mathrm{d} \tau \\
\leq & C \int_{0}^{t}\left(|m-\tilde{m}|\left|w_{x x t}\right|+|\chi-\tilde{\chi}|\left|w_{x x t}\right|+\left|w_{x x t}-\tilde{w}_{x x t}\right|\right)(x, \tau) \mathrm{d} \tau
\end{aligned}
$$

where we used Hypothesis 4.1 (i) and Proposition 2.2 (ii) and (iv). Furthermore,

$$
\begin{aligned}
& \int_{0}^{t}\left|\theta w_{x x t}-\tilde{\theta} \tilde{w}_{x x t}\right|(x, \tau) \mathrm{d} \tau \\
\leq & \int_{0}^{t}\left|w_{x x t}\right||\theta-\tilde{\theta}|(x, \tau) \mathrm{d} \tau+\int_{0}^{t}|\tilde{\theta}|\left|w_{x x t}-\tilde{w}_{x x t}\right|(x, \tau) \mathrm{d} \tau \\
\leq & \left(\int_{0}^{t}\left|w_{x x t}\right|^{2}(x, \tau) \mathrm{d} \tau\right)^{1 / 2}\left(\int_{0}^{t}|\theta-\tilde{\theta}|^{2}(x, \tau) \mathrm{d} \tau\right)^{1 / 2}+\|\tilde{\theta}\|_{\infty} \int_{0}^{t}\left|w_{x x t}-\tilde{w}_{x x t}\right|(x, \tau) \mathrm{d} \tau \\
\leq & C\left[\int_{0}^{t}\left|w_{x x t}-\tilde{w}_{x x t}\right|(x, \tau) \mathrm{d} \tau+\left(\int_{0}^{t}|\theta-\tilde{\theta}|^{2}(x, \tau) \mathrm{d} \tau\right)^{1 / 2}\right]
\end{aligned}
$$

where we used (5.3), (6.15) and the regularity part of Theorem 4.2. Similarly

$$
\int_{0}^{t}\left|w_{x x t}^{2}-\tilde{w}_{x x t}^{2}\right|(x, \tau) \mathrm{d} \tau=\int_{0}^{t}\left(\left|w_{x x t}-\tilde{w}_{x x t}\right|\left|w_{x x t}+\tilde{w}_{x x t}\right|\right)(x, \tau) \mathrm{d} \tau \leq C\left(\int_{0}^{t}\left|w_{x x t}-\tilde{w}_{x x t}\right|^{2}(x, \tau) \mathrm{d} \tau\right)^{1 / 2} .
$$


On the other hand, using again Hypothesis 4.1 (i) and Proposition 2.2 (ii),

$$
\begin{aligned}
& \frac{1}{2} \int_{0}^{t}\left|m_{t} \int_{0}^{\infty} \varphi_{m}(m, \chi, r) \mathfrak{s}_{r}^{2}\left[w_{x x}\right] \mathrm{d} r-\tilde{m}_{t} \int_{0}^{\infty} \varphi_{m}(\tilde{m}, \tilde{\chi}, r) \mathfrak{s}_{r}^{2}\left[\tilde{w}_{x x}\right] \mathrm{d} r\right| \mathrm{d} \tau \\
= & \frac{1}{2} \int_{0}^{t} \mid m_{t} \int_{0}^{\infty}\left(\varphi_{m}(m, \chi, r)-\varphi_{m}(\tilde{m}, \chi, r)\right) \mathfrak{s}_{r}^{2}\left[w_{x x}\right] \mathrm{d} r+m_{t} \int_{0}^{\infty}\left(\varphi_{m}(\tilde{m}, \chi, r)-\varphi_{m}(\tilde{m}, \tilde{\chi}, r)\right) \mathfrak{s}_{r}^{2}\left[w_{x x}\right] \mathrm{d} r \\
& +m_{t} \int_{0}^{\infty} \varphi_{m}(\tilde{m}, \tilde{\chi}, r)\left(\mathfrak{s}_{r}^{2}\left[w_{x x}\right]-\mathfrak{s}_{r}^{2}\left[\tilde{w}_{x x}\right]\right) \mathrm{d} r+\left(m_{t}-\tilde{m}_{t}\right) \int_{0}^{\infty} \varphi_{m}(\tilde{m}, \tilde{\chi}, r) \mathfrak{s}_{r}^{2}\left[\tilde{w}_{x x}\right] \mathrm{d} r \mid \mathrm{d} \tau \\
\leq & C \int_{0}^{t}\left(\left|m_{t}\right|\left(|m-\tilde{m}|+|\chi-\tilde{\chi}|+\left|w_{x x t}-\tilde{w}_{x x t}\right|\right)+\left|m_{t}-\tilde{m}_{t}\right|\right)(x, \tau) \mathrm{d} \tau .
\end{aligned}
$$

Arguing in a similar way

$$
\begin{aligned}
& \frac{1}{2} \int_{0}^{t}\left|\chi_{t} \int_{0}^{\infty} \varphi_{\chi}(m, \chi, r) \mathfrak{s}_{r}^{2}\left[w_{x x}\right] \mathrm{d} r-\tilde{\chi}_{t} \int_{0}^{\infty} \varphi_{\chi}(\tilde{m}, \tilde{\chi}, r) \mathfrak{s}_{r}^{2}\left[\tilde{w}_{x x}\right] \mathrm{d} r\right| \mathrm{d} \tau \\
= & \frac{1}{2} \int_{0}^{t} \mid \chi_{t} \int_{0}^{\infty}\left(\varphi_{\chi}(m, \chi, r)-\varphi_{\chi}(\tilde{m}, \chi, r)\right) \mathfrak{s}_{r}^{2}\left[w_{x x}\right] \mathrm{d} r+\chi_{t} \int_{0}^{\infty}\left(\varphi_{\chi}(\tilde{m}, \chi, r)-\varphi_{\chi}(\tilde{m}, \tilde{\chi}, r)\right) \mathfrak{s}_{r}^{2}\left[w_{x x}\right] \mathrm{d} r \\
& +\chi_{t} \int_{0}^{\infty} \varphi_{\chi}(\tilde{m}, \tilde{\chi}, r)\left(\mathfrak{s}_{r}^{2}\left[w_{x x}\right]-\mathfrak{s}_{r}^{2}\left[\tilde{w}_{x x}\right]\right) \mathrm{d} r+\left(\chi_{t}-\tilde{\chi}_{t}\right) \int_{0}^{\infty} \varphi_{\chi}(\tilde{m}, \tilde{\chi}, r) \mathfrak{s}_{r}^{2}\left[\tilde{w}_{x x}\right] \mathrm{d} r \mid \mathrm{d} \tau \\
\leq & C \int_{0}^{t}\left(\left|\chi_{t}\right|\left(|m-\tilde{m}|+|\chi-\tilde{\chi}|+\left|w_{x x t}-\tilde{w}_{x x t}\right|\right)+\left|\chi_{t}-\tilde{\chi}_{t}\right|\right)(x, \tau) \mathrm{d} \tau .
\end{aligned}
$$

Our aim is now to estimate $\left|m_{t}-\tilde{m}_{t}\right|$ and $\left|\chi_{t}-\tilde{\chi}_{t}\right|$ in terms of the corresponding quantities without derivatives, with the purpose to apply Gronwall's lemma. Arguing in a similar way as we did in (7.26) we deduce

$$
|\chi-\tilde{\chi}|(x, t) \leq \int_{0}^{t}\left|\chi_{t}-\tilde{\chi}_{t}\right|(x, \tau) \mathrm{d} \tau \leq C \int_{0}^{t}\left(|\theta-\tilde{\theta}|+|m-\tilde{m}|+\left|w_{x x t}-\tilde{w}_{x x t}\right|\right)(x, \tau) \mathrm{d} \tau,
$$

while, by Proposition 2.2 (ii) together with (3.12), (3.14) and (8.1),

$$
\begin{aligned}
|m-\tilde{m}|(x, t) & \leq \int_{0}^{t}\left|m_{t}-\tilde{m}_{t}\right|(x, \tau) \mathrm{d} \tau \\
& \leq C \int_{0}^{t}\left(\left|\chi_{t}-\tilde{\chi}_{t}\right|(x, \tau)+\int_{0}^{1}\left(|m-\tilde{m}|\left|w_{x x t}\right|+|\chi-\tilde{\chi}|\left|w_{x x t}\right|+\left|w_{x x t}-\tilde{w}_{x x t}\right|\right)(y, \tau) \mathrm{d} y\right) \mathrm{d} \tau .
\end{aligned}
$$

We observe that, again by (5.3) and (6.15),

$$
\int_{0}^{t} \int_{0}^{1}|m-\tilde{m}|\left|w_{x x t}\right| \mathrm{d} y \mathrm{~d} \tau \leq C \int_{0}^{t}\left(\int_{0}^{1}|m-\tilde{m}|^{2} \mathrm{~d} y\right)^{1 / 2} \mathrm{~d} \tau
$$

and in a similar way

$$
\int_{0}^{t} \int_{0}^{1}|\chi-\tilde{\chi}|\left|w_{x x t}\right| \mathrm{d} y \mathrm{~d} \tau \leq C \int_{0}^{t}\left(\int_{0}^{1}|\chi-\tilde{\chi}|^{2} \mathrm{~d} y\right)^{1 / 2} \mathrm{~d} \tau,
$$

Plugging this back into (8.3) together with (8.2) we have 


$$
\begin{aligned}
|m-\tilde{m}|(x, t) \leq & \int_{0}^{t}\left|m_{t}-\tilde{m}_{t}\right|(x, \tau) \mathrm{d} \tau \\
\leq & \left.C \int_{0}^{t}\left(|\theta-\tilde{\theta}|+|m-\tilde{m}|(x, \tau)+\left|w_{x x t}-\tilde{w}_{x x t}\right|\right)(x, \tau)+\int_{0}^{1}\left|w_{x x t}-\tilde{w}_{x x t}\right|(y, \tau) \mathrm{d} y\right) \mathrm{d} \tau \\
& +C \int_{0}^{t}\left(\int_{0}^{1}|m-\tilde{m}|^{2} \mathrm{~d} y\right)^{1 / 2} \mathrm{~d} \tau+C \int_{0}^{t}\left(\int_{0}^{1}|\chi-\tilde{\chi}|^{2} \mathrm{~d} y\right)^{1 / 2} \mathrm{~d} \tau .
\end{aligned}
$$

Then (8.2) and (8.4) imply

$$
\begin{aligned}
|m-\tilde{m}|^{2}(x, t)+|\chi-\tilde{\chi}|^{2}(x, t) \leq & C\left(\int_{0}^{t}\left(|\theta-\tilde{\theta}|^{2}+|m-\tilde{m}|^{2}+\left|w_{x x t}-\tilde{w}_{x x t}\right|^{2}\right)(x, \tau) \mathrm{d} \tau\right. \\
& \left.+\int_{0}^{t} \int_{0}^{1}\left(|m-\tilde{m}|^{2}+|\chi-\tilde{\chi}|^{2}+\left|w_{x x t}-\tilde{w}_{x x t}\right|^{2}\right)(y, \tau) \mathrm{d} y \mathrm{~d} \tau\right) .
\end{aligned}
$$

Integrating in space and exploiting Gronwall's argument we deduce

$$
\int_{0}^{1}\left(|m-\tilde{m}|^{2}+|\chi-\tilde{\chi}|^{2}\right)(y, t) \mathrm{d} y \leq C\left(\int_{0}^{1} \int_{0}^{t}\left(|\theta-\tilde{\theta}|^{2}+\left|w_{x x t}-\tilde{w}_{x x t}\right|^{2}\right)(y, \tau) \mathrm{d} y \mathrm{~d} \tau\right) .
$$

We now go back to (8.2) and (8.4), which together with the above estimate imply

$$
\begin{aligned}
|m-\tilde{m}|(x, t) \leq & \int_{0}^{t}\left|m_{t}-\tilde{m}_{t}\right|(x, \tau) \mathrm{d} \tau \leq \int_{0}^{t}\left(\left|m_{t}-\tilde{m}_{t}\right|+\left|\chi_{t}-\tilde{\chi}_{t}\right|\right)(x, \tau) \mathrm{d} \tau \\
\leq & C\left(\int_{0}^{t}\left(|\theta-\tilde{\theta}|+|m-\tilde{m}|+\left|w_{x x t}-\tilde{w}_{x x t}\right|\right)(x, \tau) \mathrm{d} \tau\right. \\
& \left.+\int_{0}^{t}\left(\int_{0}^{1}\left(|\theta-\tilde{\theta}|^{2}+\left|w_{x x t}-\tilde{w}_{x x t}\right|^{2}\right)(y, \tau) \mathrm{d} y\right)^{1 / 2} \mathrm{~d} \tau\right) .
\end{aligned}
$$

A further application of Gronwall's lemma to the term $|m-\tilde{m}|$ produces the desired inequality

$$
\begin{aligned}
& \int_{0}^{t}\left(\left|m_{t}-\tilde{m}_{t}\right|+\left|\chi_{t}-\tilde{\chi}_{t}\right|\right)(x, \tau) \mathrm{d} \tau \leq C\left(\int_{0}^{t}\left(|\theta-\tilde{\theta}|+\left|w_{x x t}-\tilde{w}_{x x t}\right|\right)(x, \tau) \mathrm{d} \tau+\right. \\
& \left.+\int_{0}^{t}\left(\int_{0}^{1}\left(|\theta-\tilde{\theta}|^{2}+\left|w_{x x t}-\tilde{w}_{x x t}\right|^{2}\right)(y, \tau) \mathrm{d} y\right)^{1 / 2} \mathrm{~d} \tau\right) .
\end{aligned}
$$

We finally observe that, by Hypothesis 4.1 (vii),

$$
\int_{0}^{t}|g(\theta, x, \tau)-g(\tilde{\theta}, x, \tau)| \mathrm{d} \tau \leq C \int_{0}^{t}|\theta-\tilde{\theta}| \mathrm{d} \tau .
$$

Now the conclusion comes arguing as in [13]: indeed we integrate in time from 0 to $t$ the difference of (4.3) for $\theta$ and $\tilde{\theta}$ testing the result by $\theta(x, t)-\tilde{\theta}(x, t)$, and then we integrate in $x$. Taking into account the above estimates we get

$$
\begin{aligned}
& \int_{0}^{1}|\theta-\tilde{\theta}|^{2}(x, t) \mathrm{d} x+\frac{1}{2} \frac{\mathrm{d}}{\mathrm{d} t} \int_{0}^{1}\left(\int_{0}^{t}\left(\theta_{x}-\tilde{\theta}_{x}\right)(x, \tau) \mathrm{d} \tau\right)^{2} \mathrm{~d} x \\
\leq & C \int_{0}^{t} \int_{0}^{1}\left(\left|w_{x x t}-\tilde{w}_{x x t}\right|^{2}+|\theta-\tilde{\theta}|^{2}\right)(x, \tau) \mathrm{d} x \mathrm{~d} \tau .
\end{aligned}
$$


Next we test the difference of the time derivatives of (4.2) for $w$ and $\tilde{w}$ by $w_{t}-\tilde{w}_{t}$, the difference of (4.1) for $u$ and $\tilde{u}$ by $w_{x x t}-\tilde{w}_{x x t}$ and sum up. The only difference with [13] is that now the Preisach operator depends also on $\chi$, thus by Hypothesis 4.1 (i) and Proposition 2.2 (ii) we get

$$
\begin{aligned}
\mid P[m, \chi, & \left.w_{x x}\right]-P\left[\tilde{m}, \tilde{\chi}, \tilde{w}_{x x}\right] \mid \\
= & \left|\int_{0}^{\infty}\left(\varphi(m, \chi, r) \mathfrak{s}_{r}\left[w_{x x}\right]-\varphi(\tilde{m}, \tilde{\chi}, r) \mathfrak{s}_{r}\left[\tilde{w}_{x x}\right]\right) \mathrm{d} r\right| \\
= & \mid \int_{0}^{\infty}\left((\varphi(m, \chi, r)-\varphi(\tilde{m}, \chi, r)) \mathfrak{s}_{r}\left[w_{x x}\right]\right. \\
& \left.+(\varphi(\tilde{m}, \chi, r)-\varphi(\tilde{m}, \tilde{\chi}, r)) \mathfrak{s}_{r}\left[w_{x x}\right] \mathrm{d} r\right) \\
& +\int_{0}^{\infty} \varphi(\tilde{m}, \tilde{\chi}, r)\left(\mathfrak{s}_{r}\left[w_{x x}\right]-\mathfrak{s}_{r}\left[\tilde{w}_{x x}\right]\right) \mathrm{d} r \mid \\
\leq & C\left(|m-\tilde{m}|+|\chi-\tilde{\chi}|+\int_{0}^{t}\left|w_{x x t}-\tilde{w}_{x x t}\right| \mathrm{d} \tau\right) .
\end{aligned}
$$

Arguing as above, after integration in space we obtain using (8.4)

$$
\begin{gathered}
\frac{1}{2} \frac{\mathrm{d}}{\mathrm{d} t} \int_{0}^{1}\left(\left(w_{t}-\tilde{w}_{t}\right)^{2}+\left(w_{x t}-\tilde{w}_{x t}\right)^{2}+\left(w_{x x}-\tilde{w}_{x x}\right)^{2}\right)(x, t) \mathrm{d} x+\int_{0}^{1}\left|w_{x x t}-\tilde{w}_{x x t}\right|^{2}(x, t) \mathrm{d} x \\
\leq C\left(\int_{0}^{1}|\theta-\tilde{\theta}|^{2}(x, t) \mathrm{d} x+\int_{0}^{t} \int_{0}^{1}\left|w_{x x t}-\tilde{w}_{x x t}\right|^{2}(x, t) \mathrm{d} x \mathrm{~d} \tau\right) .
\end{gathered}
$$

We now multiply (8.5) by $2 C$ and add the result to (8.6) to get

$$
\begin{aligned}
& \int_{0}^{1}\left(\left|w_{x x t}-\tilde{w}_{x x t}\right|^{2}+C|\theta-\tilde{\theta}|^{2}\right)(x, t) \mathrm{d} x \\
& \quad+\frac{1}{2} \frac{\mathrm{d}}{\mathrm{d} t} \int_{0}^{1}\left[2 C\left(\int_{0}^{t}\left(\theta_{x}-\tilde{\theta}_{x}\right)(x, \tau) \mathrm{d} \tau\right)^{2}+\left(w_{t}-\tilde{w}_{t}\right)^{2}+\left(w_{x t}-\tilde{w}_{x t}\right)^{2}+\left(w_{x x}-\tilde{w}_{x x}\right)^{2}\right](x, t) \mathrm{d} x \\
& \leq\left(C+2 C^{2}\right) \int_{0}^{t} \int_{0}^{1}\left(\left|w_{x x t}-\tilde{w}_{x x t}\right|^{2}+|\theta-\tilde{\theta}|^{2}\right)(x, \tau) \mathrm{d} x \mathrm{~d} \tau .
\end{aligned}
$$

Hence applying Gronwall's lemma we infer that $w=\tilde{w}, \theta=\tilde{\theta}$, and the proof of Theorem 4.2 is complete.

\section{REFERENCES}

[1] E. Bonetti and G. Bonfanti, Well-posedness results for a model of damage in thermoviscoelastic materials. Ann. Inst. Henri Poincaré 25 (2008) 1187-1208.

[2] E. Bonetti and G. Schimperna, Local existence for Frémond's model of damage in elastic materials. Continuum Mech. Therm. 16 (2004) 319-335.

[3] E. Bonetti, G. Schimperna and A. Segatti, On a doubly nonlinear model for the evolution of damaging in viscoelastic materials. J. Diff. Equ. 218 (2005) 91-116.

[4] S. Bosia, M. Eleuteri, J. Kopfová and P. Krejčí, Fatigue and phase in a oscillating plate. Physica B 435 (2014) 1-3.

[5] M. Brokate, C. Carstensen and J. Valdman, A quasi-static boundary value problem in multi-surface elastoplasticity. I. Analysis. Math. Methods Appl. Sci. 27 (2004) 1697-1710.

[6] M. Brokate, K. Dreßler and P. Krejčí, Rainflow counting and energy dissipation for hysteresis models in elastoplasticity. Euro. J. Mech. A/Solids 15 (1996) 705-735.

[7] M. Brokate and A.M. Khludnev, Existence of solutions in the Prandtl-Reuss theory of elastoplastic plates. Adv. Math. Sci. Appl. 10 (2000) 399-415.

[8] E. Davoli, T. Roubíček and U. Stefanelli, Dynamic perfect plasticity and damage in viscoelastic solids. Z. Angew. Math. Mech. 99 (2019) 1-27. 
[9] M. Eleuteri, J. Kopfová and P. Krejčí, A thermodynamic model for material fatigue under cyclic loading. Physica B 407 (2012) $1415-1416$.

[10] M. Eleuteri, J. Kopfová and P. Krejčí, Fatigue accumulation in an oscillating plate. Discrete Cont. Dynam. Syst. Ser. S 6 (2013) 909-923.

[11] M. Eleuteri, J. Kopfová and P. Krejčí, Non-isothermal cyclic fatigue in an oscillating elastoplastic beam. Comm. Pure Appl. Anal. 12 (2013) 2973-2996.

[12] M. Eleuteri, J. Kopfová and P. Krejčí, Fatigue accumulation in a thermo-visco-elastoplastic plate. Discrete Cont. Dyn. Syst., Ser. B 19 (2014) 2091-2109.

[13] M. Eleuteri, J. Kopfová and P. Krejčí, A new phase field model for material fatigue in oscillating elastoplastic beam. Discrete Cont. Dyn. Syst., Ser. A 35 (2015) 2465-2495.

[14] M. Eleuteri and J. Kopfová, On a new model for fatigue and phase transition in an oscillating elastoplastic plate. J. Diff. Equ. 265 (2018) 1839-1874.

[15] A. Flatten, Lokale und nicht-lokale Modellierung und Simulation thermomechanischer Lokalisierung mit Schädigung für metallische Werkstoffe unter Hochgeschwindigkeitsbeanspruchungen. BAM-Dissertationsreihe, Berlin (2008).

[16] M. Frémond and A. Visintin, Dissipation dans le changement de phase. Surfusion. Changement de phase irréversible. C. R. Acad. Sci. Paris Sér. II Méc. Phys. Chim. Sci. Univers Sci. Terre 301 (1985) 1265-1268.

[17] G. Friesecke, R.D. James and S. Müller, A hierarchy of plate models derived from nonlinear elasticity by gamma-convergence. Arch. Ration. Mech. Anal. 180 (2006) 183-236.

[18] D. Knees, R. Rossi and C. Zanini, A vanishing viscosity approach to a rate-independent damage model. Math. Models Methods Appl. Sci. (M3AS) 23 (2013) 565-616.

[19] P. Krejčí, Hysteresis, Convexity and Dissipation in Hyperbolic Equations. Gakuto Intern. Ser. Math. Sci. Appl. 8 (1996).

[20] P. Krejčí and J. Sprekels, Elastic-ideally plastic beams and Prandtl-Ishlinskii hysteresis operators. Math. Methods Appl. Sci. 30 (2007) 2371-2393.

[21] M. Kuczma, P. Litewka, J. Rakowski and J.R. Whiteman, A variational inequality approach to an elastoplastic plate-foundation system. Found Civil Environ. Eng. 5 (2004) 31-48.

[22] M. Liero and A. Mielke, An evolutionary elastoplastic plate model derived via Gamma convergence. WIAS Preprint No. 1583 (2010).

[23] M. Liero and T. Roche, Rigorous derivation of a plate theory in linear elastoplasticity via Gamma convergence. WIAS Preprint No. 1636 (2011).

[24] O. Millet, A. Cimetiere and A. Hamdouni, An asymptotic elastic-plastic plate model for moderate displacements and strong strain hardening. Eur. J. Mech. A Solids 22 (2003) 369-384.

[25] D. Percivale, Perfectly plastic plates: a variational definition. J. Reine Angew. Math. 411 (1990) 39-50.

[26] A. Mielke and T. Roubíček, Rate-independent damage processes in nonlinear inelasticity. Math. Models Meth. Appl. Sci. (M3AS) 16 (2006) 177-209.

[27] E. Rocca and R. Rossi, A degenerating PDE system for phase transitions and damage. Math. Models Methods Appl. Sci. (M3AS) 24 (2014) 1265-1341.

[28] T. Roubíček and U. Stefanelli, Finite thermoelastoplasticity and creep under small elastic strains. Math. Mech. Solids 24 (2019) 1161-1181.

[29] T. Roubíček and J. Valdman, Stress-driven solution to rate-independent elasto-plasticity with damage at small strains and its computer implementation. Math. Mech. of Solids 22 (2017) 1267-1287.

[30] M. Thomas and A. Mielke, Damage of nonlinearly elastic materials at small strain: existence and regularity results. $Z$. Angew. Math. Mech. 90 (2010) 88-112.

[31] S. Tsutsumi and R. Fincato, Cyclic elasticity model for fatigue with softening behaviour below macroscopic yielding. Mater. Des. 165 (2019) 107503.

[32] A. Visintin, Vol. 28 of Models of phase transitions. Progress in Nonlinear Differential Equations and Applications. Birkhäuser, Boston (1996)

[33] S-Y. Wang, L. Zhan, Z-L. Wang, Z.-N. Yin and H. Xiao, A direct approach towards simulating cyclic and non-cyclic fatigue failure of metals. Acta Mech. 228 (2017) 4325-4339. 